\title{
DEIM reduced order model constructed by hybrid snapshot simulation
}

\author{
Feng Bai $^{1} \cdot$ Yi Wang $^{1}$ (D)
}

Received: 1 May 2020 / Accepted: 24 November 2020 / Published online: 6 December 2020

(c) Springer Nature Switzerland AG 2020

\begin{abstract}
This paper presents a methodology that combines the hybrid snapshot simulation (Bai and Wang in Int J Comput Methods 18:2050029, 2020) and the discrete empirical interpolation method (DEIM) to reduce the computational cost of constructing a DEIM-based nonlinear reduced order model (ROM) while preserving its accuracy. In distinct contrast to its traditional counterpart, the time span of the hybrid snapshot simulation is divided into multiple intervals, and a majority of the intervals are simulated by local ROMs, while a fraction by the full order model (FOM). To tackle the challenge associated with limited FOM data for ROM-DEIM construction, a new approach is proposed to reconstruct and enrich the snapshot data of nonlinear model terms using solution data in ROM intervals. A formulation and procedure based on the cell-centered finite volume method (FVM) scheme are also developed to take into account two types of nonlinearities in ROM-DEIM: the componentwise and the transport-related, non-componentwise. A global ROM-DEIM is produced immediately at the end of the snapshot simulation and can be used to accelerate online simulation for different scenarios. The proposed methodology demonstrates excellent computational performance. Specifically, the computational time of the snapshot simulation is reduced by $\sim 50 \%$ without compromising ROM-DEIM accuracy (relative error less than $0.8 \%$ compared with FOM). The results prove feasibility of combining hybrid snapshot simulation and DEIM for robust and efficient ROM-DEIM development.
\end{abstract}

Keywords Hybrid snapshots computation - Nonlinear reduced order model · DEIM - SVD and iSVD · Finite volume method

\section{Introduction}

Numerical modeling and computation play a continuously increasing role in almost all engineering aspects, starting from physical phenomenon study, conceptual design, and high-fidelity analysis, to design optimization. Despite a variety of techniques developed, approaches of salient computational efficiency are still in urgent need to address specific, real-world engineering applications, where speed and resource usage requirements are critical. In this context, various reduced order modeling (ROM) techniques have been developed in the past two decades for simulating large, physics-based numerical problems on resourcelimited computing platforms. By projecting the full order model (FOM) onto a low-dimensional subspace, ROM can be solved with minimal usage of computational resources and time $[6,12,18,19,34,36,44,47-49]$.

In general, the ROM technique consists of two phases: the offline snapshot simulation for basis vectors extraction using representative model parameters; and the online verification simulation that generates ROM to compute transfer functions and field distributions using basis vectors obtained in the offline stage but with model parameters different from those in the snapshot simulation. The snapshot simulation is usually performed using the highfidelity FOM to generate snapshot data, and the projection basis vectors can be determined using the proper orthogonal decomposition (POD) of the snapshot data, one of

$\triangle$ Yi Wang, yiwang@cec.sc.edu | ${ }^{1}$ Department of Mechanical Engineering, University of South Carolina, 300 Main Street, Columbia, SC 29208, USA. 
the most widely used low-rank approximation methods for ROMs [39-41]. In POD, the singular value decomposition (SVD) or the eigenvalue decomposition (e.g., the method of snapshots [39-41]) is applied onto the entire snapshot data, and significance of the basis vectors also needs to be ranked. Therefore, it is computationally demanding in terms of both time and resource usage. The computational cost could be reduced using snapshot data selection methods that have been proposed to accelerate the simulation and basis extraction and to minimize generation of redundant FOM information. An adaptive snapshot simulation method with error estimation was studied, and the convergence analysis was also performed [1]. An adaptive snapshot simulation based on the weak form of PDEs with Galerkin projection and finite element basis was also demonstrated [43]. A parallelization technique was proposed for snapshot generation by dividing the entire snapshot data computation into multiple parallel threads, and then the snapshot simulation could be performed in multiprocessors for enhanced efficiency [46].

Hybrid modeling methods by combining FOMs and local ROMs into a streamlined numerical simulation were also reported for computational acceleration. Important criteria for FOM/ROM model switches were introduced in $[37,42]$ based on the importance of each individual singular value and the trajectory of ROM. Hybrid methods for adaptive dimension reduction and ROM construction to numerically solve partial differential equations (PDEs) efficiently were discussed in [21]. An approach for online model order reduction based on selective skip of the time steps during FOM simulation was developed for rapidly computing structural deformation [27]. Correspondingly, approaches for subspace basis updating and local ROM construction were also proposed, that is, when new FOM data become available, the orthogonal basis of the subspace is updated through rotation and dilation, and the ROM structure is modified by re-projection onto the new basis. There are many methods that can be used for basis updating, including the fast low-rank SVD, such as incremental singular value decomposition (iSVD) [8] and "Rank-One" modification [9], and the sequential POD and basis extension [11, 24-26, 28]. In particular, the rank-one modification was also adopted in online adaptive datadriven ROMs for POD basis updating [32] and adaptive interpolation [33]. The PDE models with Galerkin projection need to accommodate the continuous-time data with a discrete-time incremental POD [20]. The merits of iSVD could also be utilized for adaptive snapshot data selection to cut down the time and memory usage in POD, and the snapshot data could be selected with respect to the approximation error [30]. Despite these seminal works of the hybrid methods, research that can accelerate the snapshot simulation itself and minimize the offline computational cost has not been reported. In contrast to a specific, online verification simulation, model parameters for the snapshot simulation need to be more representative and accommodating to various scenarios that will be encountered in the verification simulation. More importantly, it is normal that snapshot data are generated exclusively by FOM simulation for enhanced quality. This is because the results obtained by FOM are regarded as the benchmark and yield the most accurate POD basis vectors of the solution subspace. Recently, our research group developed a hybrid method to accelerate the snapshot simulation while maintaining high quality of the snapshot data [5]. Specifically, the time span of the entire snapshot simulation is divided into several intervals, and each is simulated by either FOM or fast local ROM. Model switch is determined on the fly by evaluating several criteria that monitor the dominance of leading POD modes and ROM trajectory. Its key idea is that ROM can be used in place of FOM during the snapshot simulation, where data generated from FOM are redundant [38] with minimal contribution of new information to POD basis vectors.

Model order reduction for nonlinear problems is a more formidable challenge, primarily caused by low computational efficiency when approximating the nonlinear term. Several novel techniques have been proposed to tackle model nonlinearity, including the methods of reduced order basis [2], local basis update [3], and locally weighted basis [35]. A priori error estimation of the localized nonlinear ROM was also developed [14]. The GNAT method (Gauss-Newton with approximated tensors) was also presented, which minimizes the residual by Petrov-Galerkin projection and allows hyper-reduction based on the gappy POD technique [13]. Another two widely used approaches for reducing the computational load during nonlinear ROM computation are the discrete empirical interpolation method (DEIM) $[15,17]$ and the missing point estimation (MPE) [4]. The DEIM has been investigated for a variety of physical problems, including the Navier-Stokes flow [31], viscoplasticity [22], cardiac mechanics [7], and electromyographic POD-DEIM models [29]. Among these efforts, the research in [31] establishes the general treatments of DEIM to reduce both componentwise and non-componentwise nonlinear terms in ROM of transport phenomena.

The goal of the present research is to investigate the feasibility of establishing the DEIM approach in our hybrid snapshot simulation framework [5] to significantly reduce the computational cost associated with ROM-DEIM construction. Its novel aspects include: (1) the method to combine the hybrid snapshot simulation (alternating between FOM and ROM), snapshot data generation, and DEIM-based ROM construction; (2) the strategy to address the challenge associated with a limited number of FOM intervals in the hybrid simulation and poor representation 
of the subspace underlying nonlinear terms and DEIM. In DEIM, the POD modes of the nonlinear terms rather than those of solutions are used to determine the interpolation points. The FOM/ROM switch is based on the singular values and coefficients of the POD modes of FOM solution, and hence, the data are inadequate or unrepresentative for extracting accurate POD modes of nonlinear terms. Therefore, a new procedure is proposed to reconstruct and enrich snapshot data of nonlinear terms from the ROM solution in the hybrid snapshot simulation to improve accuracy of their POD modes and DEIM; and (3) the formulation to construct ROM-DEIMs in the cell-centered finite volume method (FVM) scheme along with properly devised data structures to consider both the componentwise and non-componentwise nonlinear terms that are widely encountered in physical transport phenomena.

The rest of this article is organized as follows: The methodology of the hybrid snapshot simulation (including criteria for FOM/ROM switch, SVD and iSVD for POD mode generations, and reconstruction of snapshot data for nonlinear terms) is described in Sect. 2. The DEIM algorithm in the cell-centered FVM implementation is introduced in
Sect. 3. A numerical heat transfer model including both the componentwise and non-componentwise nonlinearities is used as an example to elucidate the proposed method (FOM, ROM, and ROM-DEIM) in Sect. 4. In Sect. 5, the numerical results obtained from two case studies are discussed, and the computational performance of the present method is also evaluated. The paper concludes with a summary in Sect. 6.

\section{Methodology of hybrid snapshot simulation for ROM-DEIM construction}

In this section, we will describe the methodology to combine the hybrid computational approach in snapshot simulation and the ROM-DEIM construction using the snapshot data in snapshot simulation. The developed ROM-DEIM could be utilized in online verification simulation for the acquisition of model solutions as illustrated in Fig. 1. The entire time span of the snapshot simulation (labeled "1" in Fig. 1) is divided into multiple intervals. Each interval will be simulated by either FOM or ROM, and the
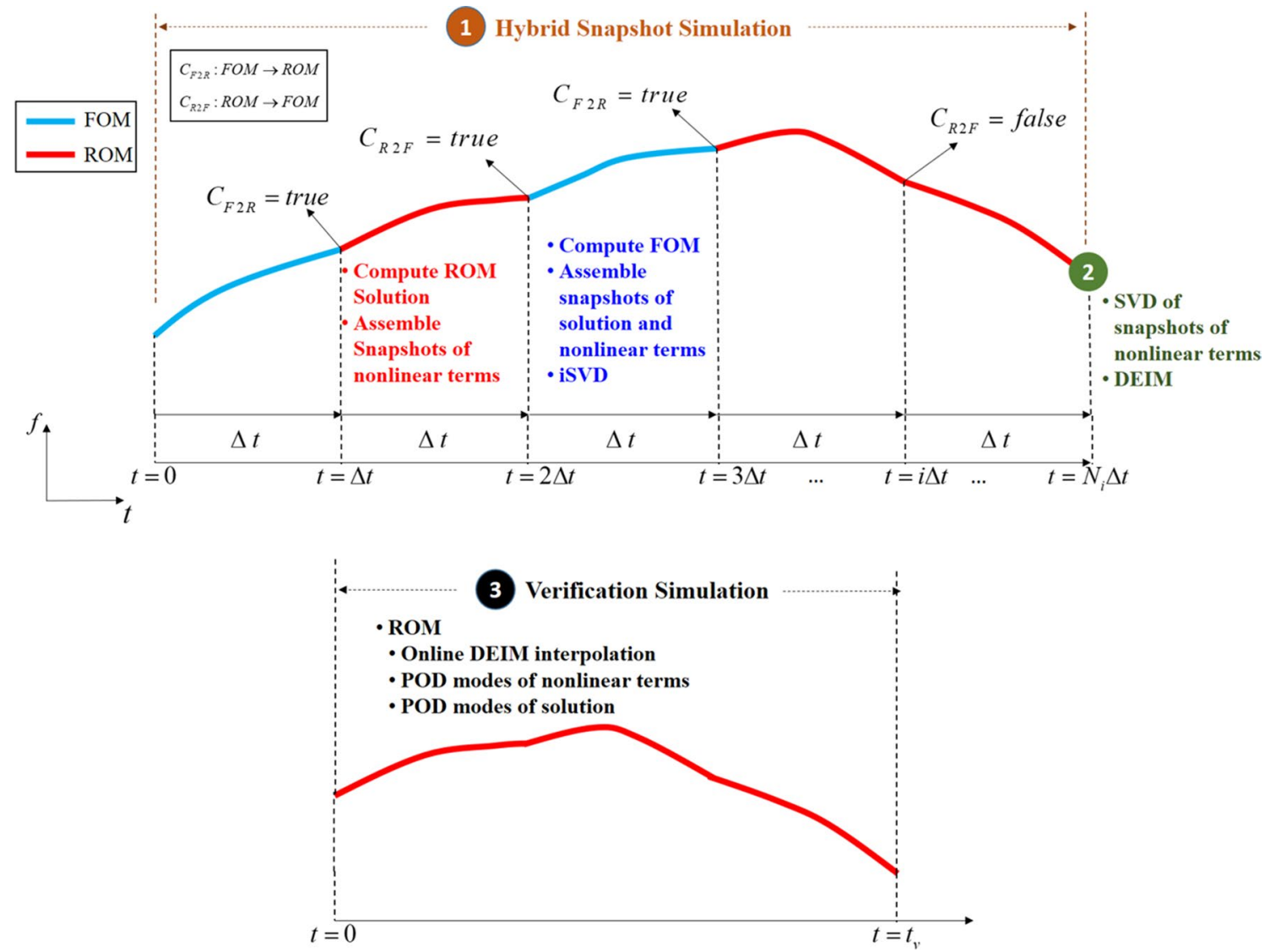

Fig. 1 The proposed methodology to construct ROM-DEIM based on hybrid snapshot simulation: (1) hybrid snapshot simulation; (2) determination of DEIM points/cells at the end of the hybrid simulation; and (3) verification simulation utilizing ROM-DEIM 
latter will be constructed and updated during the snapshot simulation, i.e., local ROM. The FOM can be written in the form of an ordinary differential equation (ODE) set:

$\frac{\mathrm{d} \mathbf{Y}(t)}{\mathrm{d} t}=\mathbf{H}(\mathbf{Y}(t))$,

where $\mathbf{Y}(t) \in \Re^{n}$ is the vector of the solution variable in the discretized computational domain, and $n$ is the dimension of the FOM solutions. $\mathbf{H} \in \mathfrak{R}^{n}$ represents the function arising from the spatial discretization of the governing equation. The corresponding form of ROM then can be written as:

$\frac{\mathrm{d} \mathbf{Y}_{r}(t)}{\mathrm{d} t}=\mathbf{U}_{r}^{T} \mathbf{H}\left(\mathbf{U}_{r} \mathbf{Y}_{r}(t)\right)$

where $\mathbf{U}_{r}$ is the truncated POD basis vector of a reduced dimension $r(r \ll n)$. The solutions of ROM are denoted as $\mathbf{Y}_{r}(t) \in \mathfrak{R}^{r}$. In each FOM interval, full solution will be computed and used as snapshot data to create and update POD modes/basis vectors $\mathbf{U}_{r}$ of the solution variable $\mathbf{Y}(t) \in \mathfrak{R}^{n}$ using the incremental singular value decomposition (iSVD). In addition, the snapshot data of the nonlinear terms will also be assembled and stored. These steps are annotated in blue in Fig. 1. In each ROM interval, the local ROM will be first built using $\mathbf{U}_{r}$ extracted in the preceding FOM interval and then computed to obtain the solution $\mathbf{Y}_{r}(t) \in \mathfrak{R}^{r}$ in the subspace, where $r$ is the dimension of the ROM solution. It is followed by reconstructing solution in the full domain $\hat{\mathbf{Y}}(t)$ and assembling the reconstructed snapshot data of the nonlinear terms (see the annotation in red in Fig. 1). The reconstructed snapshot data for the nonlinear terms will be combined and accumulated with that from the FOM interval. The rationale underlying the hybrid snapshot simulation approach is to reduce the computational complexity and cost by using the local ROMs to quickly traverse the intervals that offer negligible new information for POD mode $\mathbf{U}_{r}$ construction.

The DEIM procedure is performed at the end of the snapshot simulation, which is labeled " 2 " in Fig. 1. SVD will first be used to extract POD modes out of the accumulated snapshot data of the nonlinear terms, and DEIM will be applied onto these POD modes to determine critical interpolation points/cells. During online verification simulation (labeled " 3 " in Fig. 1), the POD modes of the solution variables, the POD modes of the nonlinear terms, and the DEIM interpolation points/cells will be utilized to assemble ROM-DEIM. The ROM-DEIM will be exclusively computed throughout the entire verification simulation. Because only a small number of the nonlinear terms at the grid points/cells will be assembled, while the rest will be interpolated during each iteration, the simulation can be run at very fast speed.
The key elements of the methodology will be discussed as follows. Specifically, the hybrid FOM/ROM simulation along with FOM/ROM switch criteria will be introduced in Sect. 2.1, SVD and iSVD in Sect. 2.2, reconstruction of the snapshot data of the nonlinear terms in Sect. 2.3. The DEIM algorithm to select the DEIM points/cells for interpolation and the procedure to assemble the nonlinear terms for ROM construction in the cell-centered FVM formulation are elucidated in detail in Sect. 3. A heat transfer model used to demonstrate the process of obtaining ROM-DEIM following the procedure above is described in Sect. 4.

\subsection{Hybrid (FOM/ROM) snapshot simulation}

For the sake of completeness of the paper, the hybrid snapshot simulation method will be briefly described in this section, and its details are reported in our previous work [5]. As discussed above, the entire snapshot simulation is divided into $N_{i}$ intervals with the equal time span $\Delta t$, and the size of the time step is $\delta t$, where $\delta t \ll \Delta t$ and $\Delta t=N \delta t ; N$ is the number of time steps in each interval. Thus, at the end of the ith time interval, the physical time traversed is $i N \delta t=i \Delta t$, and the total number of time steps simulated is $i N$. The first interval is always computed by the FOM in order to initialize the POD modes $\mathbf{U}_{r}$ using the snapshot data collected, while the simulation in the subsequent intervals will be performed using either FOM or ROM. The switch between FOM and ROM will be decided on the fly by evaluating several criteria at the end of each interval, including [5]:

(1) $\mathbf{C}_{F 2 R}$ to switch from FOM to ROM: It is evaluated at the end of the FOM interval and consists of two subcriteria $\mathbf{C}_{F 2 R, a}$ and $\mathbf{C}_{F 2 R, b}$ given by:

$$
\mathbf{C}_{F 2 R, a}=\left(\frac{\sum_{j=r_{d}+1}^{N} \sigma_{j}^{2}}{\sum_{i=1}^{N} \sigma_{i}^{2}}\right)^{\frac{1}{2}} \leq \epsilon_{a}, \mathbf{C}_{F 2 R, b}=\left(\frac{\sum_{j=r_{d}+r_{e}+1}^{N} \sigma_{j}^{2}}{\sum_{i=1}^{N} \sigma_{i}^{2}}\right)^{\frac{1}{2}} \leq \epsilon_{b} .
$$

The switch is based on importance of the POD basis vectors in terms of their contribution to snapshot data, which is determined by the singular values $\sigma_{1}, \ldots, \sigma_{j}, \ldots, \sigma_{N}$ with $j$ th singular value corresponding to the $j$ th $\mathrm{POD}$ basis vector [39-41]. $r_{d}$ is the number of basic POD modes retained, and $r_{e}$ is the extended mode. Therefore, $\mathbf{C}_{F 2 R, a}$ evaluates the ratio of the cumulative energy contained in the mode $_{d}+1 \rightarrow N$ relative to mode $1 \rightarrow N$. However, solely with $\mathbf{C}_{F 2 R, a}$ it is not possible to observe the change rate of singular values with respect to the mode number. Therefore, in order to evaluate the energy decay rate, $\mathbf{C}_{F 2 R, b}$ incorporating extended modes from $r_{d}+r_{e}+1$ to $N$ [42] is also defined. $\epsilon_{a}$ and $\epsilon_{b}$ are the tolerance parameters of the two sub-criteria. Since POD modes from $r_{d}+r_{e}+1$ to $N$ are a subset of those from $r_{d}+1$ to $N$, 
$\epsilon_{b} \leq \epsilon_{a}$ holds true. If both sub-criteria are satisfied, the FOM solution subspace can be captured very well by existing POD basis vectors. Then, the local ROM will be built and used in the next time interval.

(2) $\mathbf{C}_{R 2 F}$ to switch from ROM to FOM: It is used to determine whether the simulation should keep using ROM or switch back to FOM in the next interval. This criterion will be evaluated at the end of the local ROM interval and is given by:

$\mathbf{C}_{R 2 F}=\left(\frac{\sum_{j=r_{d}+1}^{r_{d}+r_{e}} \mathbf{Y}_{r, j}^{2}(i \Delta t)}{\sum_{j=1}^{r_{d}+r_{e}} \mathbf{Y}_{r, j}^{2}(i \Delta t)}\right)^{\frac{1}{2}} \geq \epsilon$,

where $\mathbf{Y}_{r, j}^{2}$ represents the square of the jth element of vector $\mathbf{Y}_{r}$, and the criterion represents the ratio of the two L2 norms. Note that during the ROM interval, since all the solutions move within the same POD subspace, the singular values cannot be used to define the criterion. Instead, the ROM solution vector $\mathbf{Y}_{r}(i \Delta t)$, i.e., POD modal coefficient for the extended mode from $r_{d}+1$ to $r_{d}+r_{e}$ at the end of the ith time interval is examined as a probe to evaluate their significance relative to all the modes. If $\mathbf{C}_{R 2 F}$ exceeds the tolerance $\epsilon$, it indicates that the trajectory of the ROM starts to deviate from existing POD subspace, and the simulation needs to switch back to FOM. Otherwise, POD modes are still suitable for ROM simulation in the next time interval [37]. Such FOM/ROM switch will be inspected at the end of each interval and continued until the end of the snapshot simulation.

\subsection{SVD and incremental SVD}

Generation of POD modes $\mathbf{U}_{r}$ of the solution variable $\mathbf{Y}(t)$ starts with SVD of the snapshot data produced in the initial FOM interval. Then, $\mathbf{U}_{r}$ is updated using the new snapshot data collected in each FOM interval by iSVD, but not in the ROM interval since it produces no new information to POD modes $\mathbf{U}_{r}$. At the end of the entire snapshot simulation, the ultimate POD modes $\mathbf{U}_{r}$ are obtained and could be utilized for online verification simulation. Denote $\boldsymbol{\Phi} \in \mathfrak{R}^{n \times N}$ the snapshot data collected in the initial time interval, and recall that $n$ is the dimension of the solution vector computed by FOM, and $N$ is the number of the time steps in each interval. Apply SVD onto $\boldsymbol{\Phi}$ yields:

$\boldsymbol{\Phi}=\mathbf{U} \boldsymbol{\Sigma} \mathbf{V}^{T}$

where $\mathbf{U} \in \mathfrak{R}^{n \times N}$ is the POD modal matrix containing orthnormal basis vectors of the snapshot, and $\boldsymbol{\Sigma} \in \mathfrak{R}^{N \times N}$ is a diagonal matrix with the diagonal elements $\sigma_{1}>\sigma_{2}>, \ldots,>\sigma_{i}>, \ldots,>\sigma_{N}$ sorted in the descending order along the diagonal; and $\mathbf{V} \in \Re^{N \times N}$ is also the orthonormal basis matrix. In the subsequent FOM interval that produces new snapshot data, iSVD [8] is performed to update the basis vectors in $\mathbf{U}$ through a computationally efficient procedure of low-rank SVD and basis vectors rotation. At the end of each FOM interval (except for the initial one), the existing POD matrices are updated by iSVD:

$\left[\begin{array}{ll}\mathbf{U} \boldsymbol{\Sigma} \mathbf{V}^{T} & \boldsymbol{\Phi}_{b}\end{array}\right]=\left[\begin{array}{ll}\mathbf{U} & \mathbf{J}\end{array}\right]\left[\begin{array}{ll}\boldsymbol{\Sigma} & \mathbf{L} \\ 0 & \mathbf{K}\end{array}\right]\left[\begin{array}{ll}\mathbf{V} & 0 \\ 0 & \mathbf{I}\end{array}\right]^{T} \approx[\mathbf{U} \mathbf{J}] \tilde{\mathbf{U}} \tilde{\mathbf{\Sigma}} \tilde{\mathbf{V}}^{T}\left[\begin{array}{ll}\mathbf{V} & 0 \\ 0 & \mathbf{I}\end{array}\right]^{T}$,

where $\boldsymbol{\Phi}_{b}$ is new snapshot data collected in the subsequent FOM interval; $\mathbf{J}$ is the orthonormal basis vectors spanning the orthogonal component $\mathbf{H}$ of $\boldsymbol{\Phi}_{b}$ with $\mathbf{H}=\left(\mathbf{I}-\mathbf{U U}^{T}\right) \boldsymbol{\Phi}_{b} ; \quad \mathbf{L}=\mathbf{U}^{T} \boldsymbol{\Phi}_{b} ; \quad \mathbf{K}=\mathbf{J}^{T} \mathbf{H} ;$ $\mathbf{Q}=\left[\begin{array}{ll}\boldsymbol{\Sigma} & \mathbf{L} \\ 0 & \mathbf{K}\end{array}\right] \in \mathfrak{R}^{2 N \times 2 N} ; \quad \tilde{\mathbf{U}} \in \mathfrak{R}^{2 N \times N}, \quad \tilde{\boldsymbol{\Sigma}} \in \mathfrak{R}^{N \times N}$, and $\tilde{\mathbf{V}} \in \mathfrak{R}^{2 N \times N}$ are the truncated SVD matrices generated from $\mathbf{Q}$. Then, the new POD modes are updated by $\mathbf{U} \leftarrow[\mathbf{U} \mathbf{J}] \tilde{\mathbf{U}}, \boldsymbol{\Sigma} \leftarrow \tilde{\boldsymbol{\Sigma}}, \mathbf{V} \leftarrow\left[\begin{array}{ll}\mathbf{V} & 0 \\ 0 & \mathbf{I}\end{array}\right] \tilde{\mathbf{V}}$.

During the ROM interval, the newest POD modes $\mathbf{U}$ obtained above are truncated to yield ROM projection matrix $\mathbf{U}_{r} \in \mathfrak{R}^{n \times r}$ with a lower rank of $r=r_{d}+r_{e} \ll N$, which in Matlab syntax is $\mathbf{U}_{r}=\mathbf{U}(:, 1: r)$. Then, $\mathbf{U}_{r}$ will be used for ROM construction and computation. Since the truncated dimension $r$ is much smaller than the FOM dimension $n$, the computational time and resource usage for the ROM interval are notably less than that of the FOM interval. Note that in the present work, two different sets of POD modes $\mathbf{U}$ and $\mathbf{U}_{r}$ are, respectively, used in iSVD for mode updating and ROM computation, and the latter is the subset of the former. The reason for doing this is given in [5].

\subsection{Reconstruction of snapshot data for nonlinear terms}

When nonlinear terms are present in the ROM, its computational efficiency can be appreciably compromised. As described above, various techniques have been proposed to address the issue, including the discrete empirical interpolation method-DEIM $[15,17]$. In order to perform DEIM, the snapshot data for the nonlinear terms need to be computed first, which traditionally is obtained by substituting FOM solution data into the nonlinear operators. In this section, we will describe the process to obtain the snapshot data for the nonlinear terms using the hybrid FOM and ROM solution.

The numerical model under present consideration is a typical transport equation spatially discretized by cell-centered FVM and includes two kinds of nonlinearities: (1) the inter-cell nonlinear term $\mathbf{F}(\mathbf{Y})$ that describes the transport 
flux among mesh cells, such as thermal conduction with temperature-dependent thermal conductivity; and (2) the cell-wise nonlinear term $\mathbf{G}(\mathbf{Y})$ that only involves contribution from a single cell, such as the nonlinear boundary conditions and heat sources. Thus, the governing transport equation in the ordinary differentiation equation (ODE) form is given by:

$\frac{\mathrm{d} \mathbf{Y}(t)}{\mathrm{d} t}=\mathbf{F}(\mathbf{Y}(t))+\mathbf{G}(\mathbf{Y}(t))$

Recall that $\mathbf{Y} \in \Re^{n}$ is the FOM solution vector in the whole computational domain with a dimension $n$, and $t$ is the time. Note that Eq. 7 is obtained by discretizing the spatial differential terms of the governing partial differentiation equation (PDE). If there is any constant or linear term in Eq. 7, they can be incorporated into either $\mathbf{F}(\mathbf{Y})$ or $\mathbf{G}(\mathbf{Y})$ without losing the generality of analysis as follows. To reduce computational cost, the FOM solution can be projected into a low-dimensional POD modal subspace $\mathbf{U}_{r}$ determined by the snapshot data $\boldsymbol{\Phi}$ above and is approximated by $\mathbf{Y} \approx \mathbf{U}_{r} \mathbf{Y}_{r}$, which when substituted into Eq. 7 yields:

$\frac{\mathrm{d} \mathbf{Y}_{r}(t)}{\mathrm{d} t}=\mathbf{U}_{r}^{T} \mathbf{F}\left(\mathbf{U}_{r} \mathbf{Y}_{r}(t)\right)+\mathbf{U}_{r}^{T} \mathbf{G}\left(\mathbf{U}_{r} \mathbf{Y}_{r}(t)\right)$,

where $\mathbf{Y}_{r}$ is the ROM solution vector with the dimension of $r=r_{d}+r_{e} \ll n$. Equation 8 clearly reveals the root for low efficiency of ROM caused by the nonlinear terms. That is, the ROM solution $\mathbf{Y}_{r}$ needs to be first reconstructed in the full domain by $\hat{\mathbf{Y}}=\mathbf{U}_{r} \mathbf{Y}_{r}$, and then the nonlinear terms are assembled in the full domain using $\hat{\mathbf{Y}}$. To tackle the limitation, DEIM accelerates assembly of the vectorized nonlinear terms by identifying their POD modal subspace and then interpolating to obtain all their entries using a smaller number of selected entries at important mesh cells. Accordingly, the snapshot data of the nonlinear terms $\mathbf{F}$ and $\mathbf{G}$ (rather than the solution variable $\mathbf{Y}$ ) need to be collected to extract their POD modes and undertake DEIM.

Traditionally, the snapshot data for the nonlinear terms are computed using FOMs only [11, 15, 23, 43] because of the demanding requirement for data quality and basis vector representation. Specifically, the FOM solution $\mathbf{Y}(t)$ is obtained at the end of the snapshot simulation, and then substituted into the nonlinear operators $\mathbf{F}(\mathbf{Y}(t))$ and $\mathbf{G}(\mathbf{Y}(t))$ on the RHS of Eq. 7. However, in our hybrid method, only a few intervals are computed by FOM during the snapshot simulation, leading to a limited number of snapshot data and inadequate presentation of POD subspace of the nonlinear terms. The criteria to determine FOM/ROM switch above are based on the singular values and ROM trajectory of the solution variable $\mathbf{Y}$. Therefore, the solution data generated in the FOM interval in general do not represent well the subspace of nonlinear terms. In addition, DEIM selects interpolation points in the spatial domain of computational meshes. For large models, a large number of DEIM interpolation points may be required, and the snapshot data collected in the FOM interval may be insufficient for DEIM. To address the issue, a new approach is proposed in this paper, which includes two steps: (1) at the end of each time step in FOM interval, e.g., the ith interval, following the traditional method, the FOM solution is substituted into the nonlinear operators to yield the snapshot data of the nonlinear terms, i.e.,

$\mathbf{N}_{F}^{i}=\mathbf{F}(\mathbf{Y}(\boldsymbol{\tau})) \in \mathfrak{R}^{n \times N}, \mathbf{N}_{G}^{i}=\mathbf{G}(\mathbf{Y}(\boldsymbol{\tau})) \in \mathfrak{R}^{n \times N}$,

where $\tau=(i-1) \Delta t+[\delta, 2 \delta, \ldots, j \delta, N \delta]$ is all the time instances in the $i$ th interval and signifies concatenating their solution data. Hereafter, $\mathbf{N}$ is used to denote the snapshot data of the nonlinear terms to distinguish from that of the FOM solutions $\boldsymbol{\Phi}$; and (2) at the end of each time step in ROM interval, the approximate solutions in the full domain are first reconstructed by $\hat{\mathbf{Y}}=\mathbf{U}_{r} \mathbf{Y}_{r}$, which is then used to assemble the nonlinear terms and create their snapshot data,

$\hat{\mathbf{N}}_{F}^{i}=\mathbf{F}\left(\mathbf{U}_{r} \mathbf{Y}_{r}(\boldsymbol{\tau})\right), \hat{\mathbf{N}}_{G}^{i}=\mathbf{G}\left(\mathbf{U}_{r} \mathbf{Y}_{r}(\boldsymbol{\tau})\right)$,

where $\wedge$ denotes the quantities from or reconstructed from the ROM solution. In other words, $\hat{\mathbf{N}}_{F}^{i}$ and $\hat{\mathbf{N}}_{G}^{i}$ are the snapshot data for the nonlinear terms obtained by applying the nonlinear operators onto the approximate solutions $\hat{\mathbf{Y}}$ in the full domain.

The procedure above is then repeated for each FOM or ROM interval to obtain the snapshot data of the nonlinear terms in the entire time span of the hybrid snapshot simulation, which are assembled into a single matrix $\mathbf{N}_{F}(\mathbf{Y}(t))$ and $\mathbf{N}_{G}(\mathbf{Y}(t))$. Note that $\mathbf{N}_{F}$ and $\mathbf{N}_{G}$ without superscript $i$ denote the snapshot data of the entire snapshot simulation for the two nonlinear parts $\mathbf{F}$ and $\mathbf{G}$, respectively. Fig. 2 illustrates the procedure to assemble snapshot data for nonlinear terms, and recall that $N_{i}$ is the total number of the time intervals used in the simulation. The parts in orange and in blue represent the snapshot data from the FOM solutions and the reconstructed ROM solutions, respectively.

\section{DEIM and cell-centered FVM implementation}

In this section, the DEIM algorithm and its implementation in cell-centered FVM formulation will be described. 
Fig. 2 The procedure to assemble snapshot data for nonlinear terms using a mix of FOM and reconstructed ROM solutions from the hybrid snapshot simulation

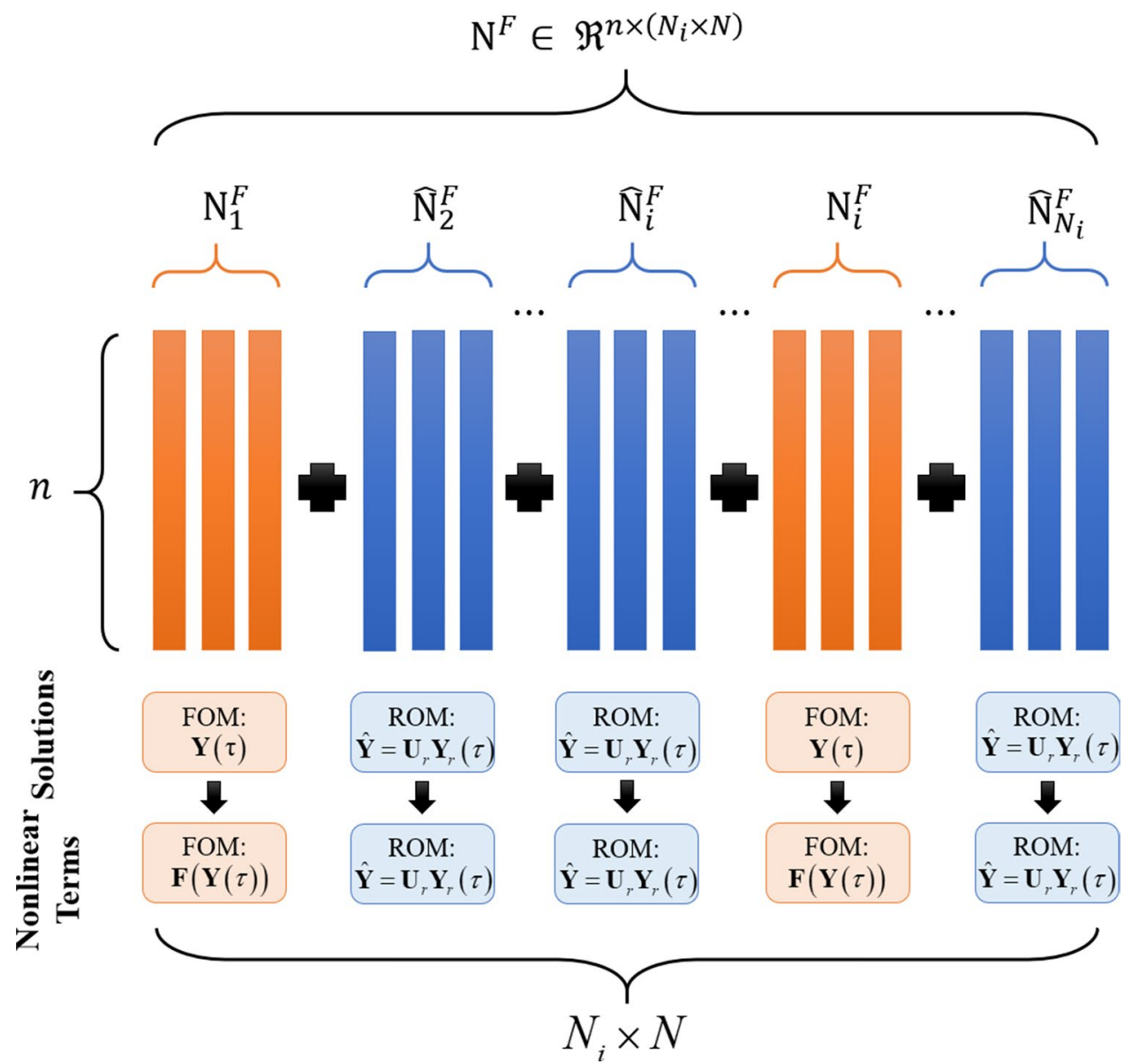

\subsection{DEIM algorithm}

DEIM was developed to reduce the cost of assembling nonlinear terms in ROMs $[15,17]$ and includes three key steps: (1) taking POD of the entire snapshot data of the nonlinear terms, which is performed by SVD at the end of the hybrid snapshot simulation in this paper in order to preserve high accuracy; (2) selecting important points/cells (i.e., their indices) that correspond to the entry with the largest residual of reconstructing the next basis vector in POD modes $[15,17]$; and (3) assembling the nonlinear terms only at the selected points/cells, which are then used to interpolate the rest nonlinear terms in the whole computational domain. Note that steps 1 and 2 are undertaken at the end of the hybrid snapshot simulation, and step 3 is during the online verification simulation for computational acceleration.

Specifically, SVD is first applied to mixed snapshot data of the nonlinear terms $\mathbf{N}_{F}$ and $\mathbf{N}_{G}$ above separately to generate POD modes $\mathbf{U}_{F}$ and $\mathbf{U}_{G}$ with the low dimensions:

$\mathbf{N}_{F} \approx \mathbf{U}_{F} \boldsymbol{\Sigma}_{F} \mathbf{V}_{F}^{T}$

$\mathbf{N}_{G} \approx \mathbf{U}_{G} \boldsymbol{\Sigma}_{G} \mathbf{V}_{G}^{T}$.
Note that subscripts $F$ and $G$ are employed to distinguish from the POD modes $\mathbf{U}_{r}$ of the FOM solution above. POD matrices for both are truncated at the dimension $m_{F}$ and $m_{G}$ and span the dominant low-dimensional subspace of the nonlinear terms, $\mathbf{F}$ and $\mathbf{G}$, respectively. Correspondingly, their truncated POD modes have the size of $\mathbf{U}_{F} \in \Re^{n \times m_{F}}$ and $\mathbf{U}_{G} \in \Re^{n \times m_{G}}$.

In the DEIM algorithm [15], a matrix $\mathbf{P} \in \mathfrak{R}^{n \times m}$ is defined to select the interpolation indices (i.e., grid points/cells) in a $n$ dimensional system. Denote $m$ the number of the selected indices, the $\mathbf{P}$ matrix can be expressed by:

$\mathbf{P}=\left[\mathbf{e}_{\wp_{1}}, \mathbf{e}_{\wp_{2}}, \ldots, \mathbf{e}_{\wp_{m}}\right]$,

where $\mathbf{e}_{\wp_{i}}=[0, \ldots, 0,1,0, \ldots, 0]^{T}$ is the $\wp_{i}$ th column of the identity matrix $\mathbf{I}_{n} \in \mathfrak{R}^{n \times n}$ with $n$ dimension for $i=1, \ldots, m$. In our model, two different selection matrices $\mathbf{P}_{F}$ and $\mathbf{P}_{G}$ with the rank of $m_{F}$ and $m_{G}$, respectively, are used for DEIM approximation of the inter-cell term $\mathbf{F}\left(\mathbf{U}_{r} \mathbf{Y}_{r}(t)\right)$ and the cell-wise nonlinear term $\mathbf{G}\left(\mathbf{U}_{r} \mathbf{Y}_{r}(t)\right)$ in Eq. 8, $[15,17]$ for verification simulation: 
$\mathbf{F}\left(\mathbf{U}_{r} \mathbf{Y}_{r}(t)\right) \approx \mathbf{U}_{F}\left(\mathbf{P}_{F}^{T} \mathbf{U}_{F}\right)^{-1} \mathbf{P}_{F}^{T} \mathbf{F}\left(\mathbf{U}_{r} \mathbf{Y}_{r}(t)\right)$,

$\mathbf{G}\left(\mathbf{U}_{r} \mathbf{Y}_{r}(t)\right) \approx \mathbf{U}_{G}\left(\mathbf{P}_{G}^{T} \mathbf{U}_{G}\right)^{-1} \mathbf{P}_{G}^{T} \mathbf{G}\left(\mathbf{U}_{r} \mathbf{Y}_{r}(t)\right)$.

The interpolation indices $\wp_{1}, \ldots, \wp_{m_{F}}$ in $\mathbf{P}_{F}$ and $\wp_{1}, \ldots, \wp_{m_{G}}$ in $\mathbf{P}_{G}$ are selected by the DEIM algorithm in [15].

Equation 13 implies two means to accelerate computation during online verification simulation: first, $\mathbf{P}_{F}^{T}$ and $\mathbf{P}_{G}^{T}$ left multiplied to $\mathbf{F}$ and $\mathbf{G}$ essentially indicate that only $m_{F}$ and $m_{G}$ rows of $\mathbf{F}$ and $\mathbf{G}$ need to be assembled; and second, the following matrices remain the same during online simulation and can be precomputed to further cut down the computational cost:

$\mathbf{C}_{F}=\mathbf{U}_{F}\left(\mathbf{P}_{F}^{T} \mathbf{U}_{F}\right)^{-1}, \mathbf{C}_{G}=\mathbf{U}_{G}\left(\mathbf{P}_{G}^{T} \mathbf{U}_{G}\right)^{-1}$.

This is because during verification simulation, the quantities associated with DEIM, such as $\mathbf{P}_{F}^{T}, \mathbf{P}_{G^{\prime}}^{T} \mathbf{U}_{F}$, and $\mathbf{U}_{G}$ will remain the same (and will be obtained at the end of the snapshot simulation). In the next section, we will describe the procedure to assemble both the inter-cell and the cellwise nonlinear terms in the cell-centered FVM formulation, both of which need to be updated during online verification simulation that poses a challenge for efficient ROM construction and will be addressed by DEIM.

\subsection{Nonlinear terms updating}

As shown in Eq. 8, the RHS of the ROM consists of two kinds of nonlinear terms, the inter-cell $\mathbf{F}\left(\mathbf{U}_{r} \mathbf{Y}_{r}(t)\right)$ and the cell-wise $\mathbf{G}\left(\mathbf{U}_{r} \mathbf{Y}_{r}(t)\right)$. The latter is caused by nonlinear phenomena that do not involve transport between two cells, e.g., the nonlinear heat source within the cell or from boundaries. However, $\mathbf{F}\left(\mathbf{U}_{r} \mathbf{Y}_{r}(t)\right)$ is contributed by the transport flux between two connected cells in the computational domain (normalized by the mass or capacitance term associated with the temporal derivative, see Sect. 4), such as the heat flux through thermal conduction. The flux between a cell $P$ and its adjacent cell $J$ is written as:

$\boldsymbol{\Psi}=-\alpha_{j}\left(Y_{J}-Y_{P}\right)$,

where $P$ is the index of the present cell, on which the thermal balance needs to be established; $Y$ is the value of the solution variable at the center of cell $J$ and $P$, and $j$ is the index of the face between cell $P$ and cell $J$. As shown in Fig. 3 for an FVM model in 2D, $J$ can be $E, S, W$, and $N$ depending on the position of the adjacent cell relative to the present cell; and $j$ can take $e, s, w$, and $n$ representing the face linking cell $P$ and its adjacent cells. $\alpha_{j}=(k A / \delta)_{j}$ is the transport link at the cell face $j$, where $A$ and $\delta$ are, respectively, the area of the face and the distance between cell $P$ and its adjacent cell $J$, and $k$ is the transport

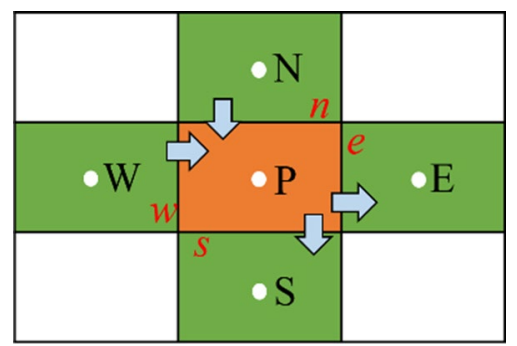

Fig. 3 Finite volume method (FVM)-based representation of FOM in a $2 \mathrm{D}$ computational domain

coefficient (e.g., thermal conductivity in heat transfer) that gives rise to the model nonlinearity if depending on $Y$, and $Y_{p}$.

The computation of the inter-cell nonlinear term $\mathbf{F}\left(\mathbf{U}_{r} \mathbf{Y}_{r}(t)\right)$ is demanding and involves multiple steps, including (1) reconstructing the solution in the full domain $\mathbf{U}_{r} \mathbf{Y}_{r}(t) ;(2)$ updating variable-dependent transport coefficient $k$ at the face between cells; and (3) assembling $\mathbf{F}$ terms by establishing the flux balance for each computational cell using Eq. 15. Steps (1)-(3) need to be performed for each iteration in each time step, which slows down the ROM simulation. To reduce the computational loads, the DEIM method discussed above will be utilized. As indicated by Eq. 13, once the POD modes $\mathbf{U}_{F}$ and the interpolation indices $\mathbf{P}_{F}$ of the nonlinear term are available, only entries at DEIM interpolation points, i.e., $\mathbf{P}_{F}^{T} \mathbf{F}\left(\mathbf{U}_{r} \mathbf{Y}_{r}(t)\right)$ rather than the entire vector $\mathbf{F}$ need to be assembled, and the rest entries can be interpolated. Although DEIM can determine those cells for interpolation (which hereafter is termed DEIM cell or interpolation cell as shown in Fig. 4a), $\mathbf{F}\left(\mathbf{U}_{r} \mathbf{Y}_{r}(t)\right)$ arises from the inter-cell transport flux, and therefore, the adjacent cells of the DEIM cells also need to be extracted in order to calculate the transport flux across their interfaces(red lines in Fig. $4 \mathrm{~b}$ and $\mathrm{c}$ ) and assemble the entries in $\mathbf{F}\left(\mathbf{U}_{r} \mathbf{Y}_{r}(t)\right)$ at the DEIM cells. The retrieval of the adjacent cells can be accomplished by constructing two data structures during numerical model initialization: the cell-to-face data structure and the face-to-cell data structure as shown in Fig. 5.

Figure 5 a illustrates the cell-to-face data structure for a 2D computational domain with $n$ quadrilateral cells. It takes the index of the cell as the entry (the first column) and retrieves the indices of the four faces surrounding the cell (the second-fifth column). As shown for the Ith and the $m$ th cell, the face indices $I_{1} \sim I_{4}$, and $m_{1} \sim m_{4}$ are, respectively, extracted. Figure $5 \mathrm{~b}$ illustrates the corresponding face-to-cell data structure with $n f$ faces in total, which takes the index of the face as the entry (the first column) and extracts the indices of the two cells on both sides of the face since each face connects two cells. For example, the cell indices $l_{1} \sim I_{2}$ and $m_{1} \sim m_{2}$ 
Fig. 4 The procedure for identifying the $\mathbf{a}$ DEIM cells, $\mathbf{b}$ surrounding faces (red lines) and $\mathbf{c}$ adjacent cells (Ad) of the DEIM cells in a 2D computational domain

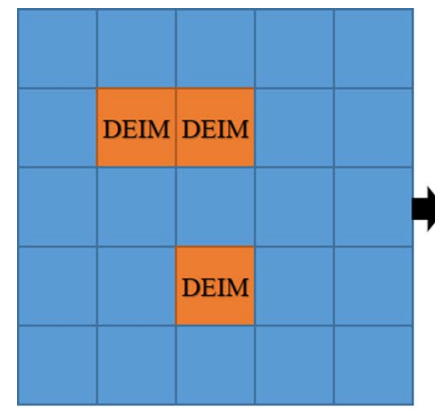

(a)

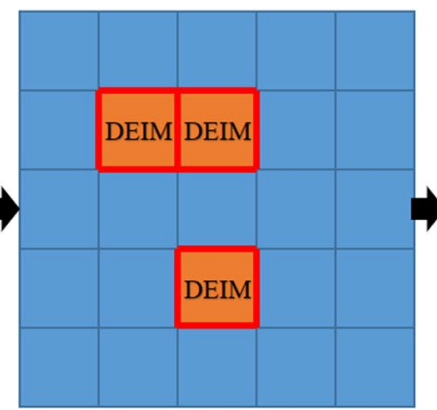

(b)

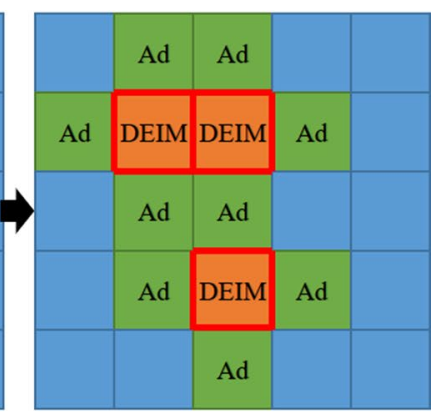

(c)

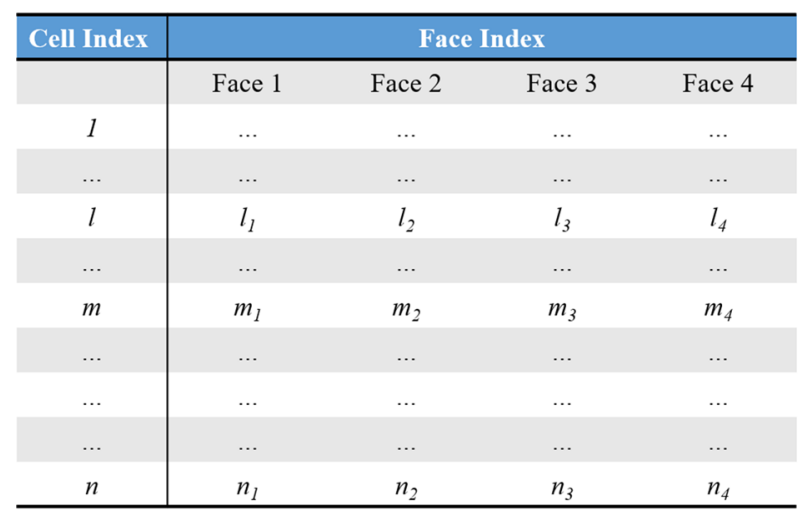

(a)

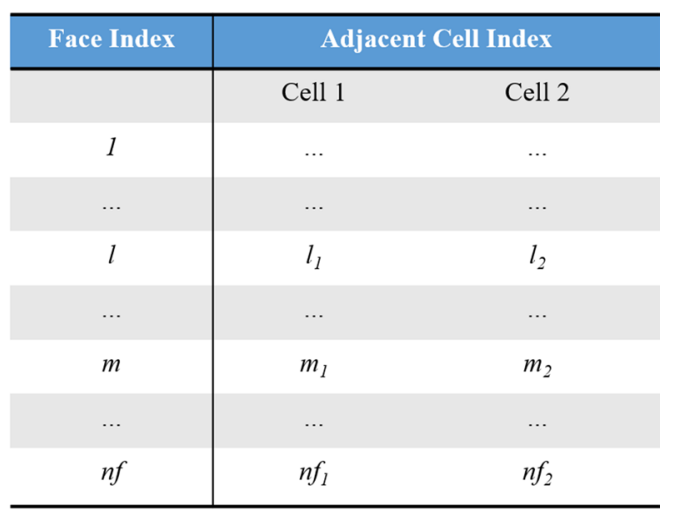

(b)

Fig. 5 The data structure used for nonlinear terms assembly at the DEIM cells: a cell-to-face and $\mathbf{b}$ face-to-cell

are, respectively, extracted for the /th and the $m$ th face. With both data structures, we then can follow the procedure in Fig. 4 to determine all the adjacent cells, which start with the DEIM algorithm presented in Sect. 3.1 to determine the DEIM cells (Fig. 4a). Then cell-to-face data structure will be queried using the indices of the DEIM cells as the entry to determine their surrounding surfaces, i.e., red lines in Fig. $4 \mathrm{~b}$. Note that the face indices retrieved may be repeated because two DEIM cells could be neighboring to each other, such as the two DEIM cells at the top of Fig. 4b. This can be resolved by eliminating the redundant indices, e.g., using the function 'unique' in Matlab or other equivalents. Then, the indices of the surround faces are entered into the face-to-cell data structure to extract indices of all cells on both sides of those faces, which include the DEIM cell and their adjacent cells. Again the retrieved cell indices may be redundant for several reasons: Two DEIM cells are next to each other (e.g., the two DEIM cells at the top of Fig. 4c) and one cell is adjacent to two DEIM cells (e.g., the cell at the center of Fig. 4c adjacent to the two DEIM cells above and below). Likewise, those repeated cell indices can be eliminated easily. In summary, at the end of the DEIM, three sets of indices will be kept for online verification simulation, which are the DEIM cells $\mathbf{P}_{F}$, the surrounding face $\mathbf{I}_{f}$, and the combined DEIM and adjacent cells $\mathbf{I}_{c^{\prime}}$ and the size of $\mathbf{P}_{F}$ is smaller than $\mathbf{I}_{c}$. Again $\mathbf{I}_{c}$ is used to obtain the values of variables $\mathbf{Y}$ at all DEIM and adjacent cells for computing the transport fluxes across the surrounding faces with indices $\mathbf{I}_{f}$. The transport fluxes are then used to assemble the entries with indices $\mathbf{P}_{F}$ in $\mathbf{F}\left(\mathbf{U}_{r} \mathbf{Y}_{r}(t)\right)$. Note that for the cell-wise nonlinear term $\mathbf{G}\left(\mathbf{U}_{r} \mathbf{Y}_{r}(t)\right)$, only indices $\mathbf{P}_{G}$ are needed. More detailed information about approximation of the transport phenomenon could be found in [13] (for finite volume) and in [15](for finite difference). When the second- or higherorder spatial discretization is employed, neighbours of the DEIM cells need to be extracted to construct the governing equation of the DEIM cell. Thus, the process to identify neighboring cells will be performed twice or multiple times with the first one to search for the neighbors of the DEIM cells, and the subsequent ones to identify the neighbors of neighbors. The duplicated cell indices will be eliminated at the end of the search for enhanced DEIM approximation accuracy. In the next section, the entire process will be described using the heat transfer as an example problem. 


\section{Numerical model of heat transfer}

The numerical model of heat transfer based on FVM will be presented in this section and used for verifying ROM-DEIM obtained by the hybrid snapshot simulation method. We will first describe FOM and ROM without DEIM used in the offline hybrid snapshot simulation, and then introduce ROM-DEIM that is constructed at the end of the snapshot simulation and used for online verification simulation.

\subsection{FOM and ROM}

The heat transfer model presented in this paper consists of different materials and involves the thermal conduction at the interior of the computational domain, and the prescribed heat flux and radiation (and convection as needed) at the boundaries. The thermal conductivity is temperature dependent, and therefore, introduces nonlinearity to the inter-cell transport term $\mathbf{F}$. The cell-wise nonlinear term $\mathbf{G}$ arises from thermal contributions at boundaries, such as applied heat flux and radiation. The governing equation of the heat transfer in the chip can be written as:

$\rho C_{p} \frac{\mathrm{d} T}{\mathrm{~d} t}=\nabla \cdot(k(T) \nabla T)+Q$,

where $T$ is the scalar temperature; $\rho$ is the density; $k$ and $C_{p}$ are, respectively, the temperature-dependent thermal conductivity and specific heat of the constitute materials; and $Q$ is the heat source term. The spatial differential of the thermal governing Eq. 16 is discretized by FVM following the schematic in Fig. 3, yielding [45]:

$$
\begin{aligned}
\rho C_{p} & V_{p} \frac{\mathrm{d} T_{P}}{\mathrm{~d} t} \\
= & -\left(\alpha_{e}+\alpha_{s}+\alpha_{w}+\alpha_{n}\right) T_{P}+\alpha_{e} T_{E}+\alpha_{s} T_{s} \\
& +\alpha_{w} T_{W}+\alpha_{n} T_{N}+Q_{P} V_{P}+A q_{b}+R_{s}\left(T_{P}^{4}-T_{\infty, R}^{4}\right) \\
= & -\alpha_{p} T_{P}+\alpha_{e} T_{E}+\alpha_{s} T_{s} \\
& +\alpha_{w} T_{W}+\alpha_{n} T_{N}+Q_{P} V_{P}+A q_{b}+R_{s}\left(T_{P}^{4}-T_{\infty, R}^{4}\right)
\end{aligned}
$$

where $V_{p}$ is the volume of the cell $P ; \alpha_{j}$ is the conductive transport link at the cell face $j$ (see Sect. 3.2); $a_{p}=a_{e}+a_{s}+a_{w}+a_{n} ; a_{b}$ is the heat flux applied at boundaries; $A$ is the area of the face of the boundary cell where the heat flux is applied, and $R_{s}=A \sigma_{r}$ is the radiative links between the present cell and the far field temperature, and $\sigma_{r}$ denotes the Stefan-Boltzmann Constant. Note that for the interior cells, the last two terms in Eq. 17 for boundary conditions are not available.
Following FVM-based discretization of the spatial differential terms in the governing equation, FOM in the form of ODE is [45]:

$\mathbf{M} \frac{\mathrm{d} \mathbf{T}}{\mathrm{d} t}=\tilde{\mathbf{A}}_{n}(\mathbf{T}) \mathbf{T}+\tilde{\mathbf{R}}_{s}\left(\mathbf{T}_{\infty, R}^{* 4}-\mathbf{T}^{* 4}\right)+\tilde{\mathbf{B}}_{c}+\tilde{\mathbf{B}} \mathbf{u}$,

where $\mathbf{T} \in \mathfrak{R}^{n}$ is the vector of the temperature solution in the full computational domain with the dimension of $n ; *$ on the superscript denotes the elements-wise operation; $\mathbf{M} \in \mathfrak{R}^{n \times n}$ is the diagonal matrix, and its diagonal is populated with the thermal capacitance/mass of all computational cells; $\tilde{\mathbf{A}}_{n}(\mathbf{T}) \in \mathfrak{R}^{n \times n}$ stores the thermal conductance among cells and at the isothermal boundaries. Specifically, its element at $(i, j)$ location is contributed by the thermal conductance between the ith and the jth cell, and the thermal conductance between the ith cell and its adjacent isothermal boundary will be added onto the ith diagonal element. Since the thermal conductivities are temperature dependent, the term $\tilde{\mathbf{A}}_{n}(\mathbf{T}) \mathbf{T}$ is a nonlinear term. $\tilde{\mathbf{R}}_{s} \in \mathfrak{R}^{n \times n}$ is another diagonal matrix to describe the external radiation, and its diagonal will be populated with the external radiative heat transfer coefficient at the boundaries. Note that along the diagonal of $\tilde{\mathbf{R}}_{s}$, only the locations corresponding to the cells adjacent to the radiation boundaries will have nonzero values. $\mathbf{T}_{\infty, R} \in \mathfrak{R}^{n}$ is the environmental temperature values at the far field for the external radiation heat transfer. $\tilde{\mathbf{B}}_{c} \in \mathfrak{R}^{n}$ is the constant source term. $\tilde{\mathbf{B}} \in \mathfrak{R}^{n \times d}$ is the matrix converting the contribution from the time-dependent input vector $\mathbf{u} \in \mathfrak{R}^{d}$ to each cell, and $d$ is the number of different inputs. In this paper, $\mathbf{u}$ is the time-dependent heat flux applied at the external boundaries of the model. Although the present method is developed using finite volume discretization, it could also be formulated and solved with the FEM approach, which possesses optimality properties and may be more favorable for solving parabolic equations.

The FOM in Eq. 18 can be written in a concise form as:

$\frac{\mathrm{d} \mathbf{T}}{\mathrm{d} t}=\mathbf{A T}(t)+\mathbf{R T}(t)^{* 4}+\mathbf{D}+\mathbf{B u}$,

where $\mathbf{A}, \mathbf{R}, \mathbf{D}$, and $\mathbf{B}$ are obtained by combining several terms in Eq. 18, viz.

$$
\begin{aligned}
& \mathbf{A}=\mathbf{M}^{-1} \tilde{\mathbf{A}}_{n \prime} \\
& \mathbf{R}=\mathbf{M}^{-1} \tilde{\mathbf{R}}_{s^{\prime}} \\
& \mathbf{D}=\mathbf{M}^{-1}\left(\tilde{\mathbf{R}}_{s} \mathbf{T}_{\infty, R}^{* 4}+\tilde{\mathbf{B}}_{c}\right), \\
& \mathbf{B}=\mathbf{M}^{-1} \tilde{\mathbf{B}} .
\end{aligned}
$$

AT $(t)$ captures the dependence of internal thermal conduction and the external convection on the temperature and forms the inter-cell nonlinear term $\mathbf{F}(\mathbf{T}(t))$ in Eq. 7. The cell-wise nonlinear term $\mathbf{G}(\mathbf{T}(t))$ consists of three parts: 
the radiation term $\mathbf{R T}(t)^{* 4}$, the term $\mathbf{D}$ incorporating far field conditions for external radiation and constant source terms, and the term Bu associated with target inputs. The thermal capacitance/mass matrix is diagonal, and $\mathbf{M}^{-1}$ in Eq. 20 represents the normalization of each individual equation in Eq. 18 by the corresponding thermal mass/ capacitance of that equation. Therefore, $\mathbf{M}^{-1}$ is simply obtained by replacing diagonal elements of $\mathbf{M}$ with their reciprocals without need for matrix factorization. Note that in this paper, each individual nonlinear term is treated separately rather than being combined together following $[10,16]$.

Local ROM utilized in the hybrid snapshot simulation is obtained by projecting FOM onto the subspace spanned by the POD basis $\mathbf{U}_{r}$ with the dimension $r$, i.e., $\mathbf{T}(t) \approx \mathbf{U}_{r} \mathbf{T}_{r}(t)$. Note that no DEIM is applied to ROM during the snapshot simulation in order to maintain high quality of the snapshot data:

$\frac{\mathrm{d} \mathbf{T}_{r}(t)}{\mathrm{d} t}=\mathbf{A}_{r} \mathbf{T}_{r}(t)+\mathbf{R}_{r}\left(\mathbf{U}_{r} \mathbf{T}_{r}(t)\right)^{* 4}+\mathbf{D}_{r}+\mathbf{B}_{r} \mathbf{u}$,

where $\mathbf{T}_{r} \in \mathfrak{R}^{r}$ is the solution in the reduced domain.

$$
\begin{aligned}
& \mathbf{A}_{r}=\mathbf{U}_{r}^{T} \mathbf{A} \mathbf{U}_{r} \in \mathfrak{R}^{r \times r}, \\
& \mathbf{R}_{r}=\mathbf{U}_{r}^{T} \mathbf{R} \in \mathfrak{R}^{r \times n}, \\
& \mathbf{D}_{r}=\mathbf{U}_{r}^{T} \mathbf{D} \in \mathfrak{R}^{r}, \\
& \mathbf{B}_{r}=\mathbf{U}_{r}^{T} \mathbf{B} \in \mathfrak{R}^{r \times d} .
\end{aligned}
$$

For our heat transfer model, the thermal conductance matrix A depends on the thermal conductivity that varies with temperature values at the present and the adjacent cells. Therefore, $\mathbf{A}_{r}$ in Eq. 21 is also temperature dependent and cannot be precomputed prior to the online verification simulation. As discussed above in Sect. 3.2, its computation requires multiple steps, which for the numerical heat transfer example includes reconstructing the solution in the full domain $\mathbf{T}(t) \approx \mathbf{U}_{r} \mathbf{T}_{r}(t)$; computing the thermal conductivity and the conductance at the face, assembling the thermal conductance matrix $\mathbf{A}$, and projecting $\mathbf{A}$ onto $\mathbf{U}_{r}$ to obtain its reduced counterpart $\mathbf{A}_{r}$.

\subsection{ROM-DEIM for online verification simulation}

Updating and assembling the inter-cell transport matrix $\mathbf{A}_{r}$ in ROM above need to be performed for all computational cells in each iteration loop and each time step, which is computationally demanding and time consuming. In fact, even for the cell-wise nonlinear terms, the solution reconstruction and assembly in the full domain need to be carried out. Therefore, ROM-DEIM will be applied in the verification simulation to achieve further acceleration. Following the procedure presented in Sect. 3.1, the data in the FOM interval and the data reconstructed in the ROM interval are combined to form the snapshot data $\mathbf{N}_{A}, \mathbf{N}_{R}$, and $\mathbf{N}_{B}$ for the nonlinear terms $\mathbf{A T}(t), \mathbf{R T}(t)^{* 4}$, and $\mathbf{B u}$, respectively. Note that $\mathbf{D}$ is a constant term in the present study, and DEIM will not be applied onto it. Then, their individual POD modes can be computed using SVD according to Eq. 11, yielding $\mathbf{U}_{A} \in \Re^{n \times m_{A}}, \mathbf{U}_{R} \in \Re^{n \times m_{R}}$, and $\mathbf{U}_{B} \in \Re^{n \times m_{B}}$, respectively, where $m_{A}, m_{R}$, and $m_{B}$, are the number of POD modes kept and also the number of the DEIM cells for interpolation. Thus, the DEIM interpolation matrices for each nonlinear term can be precomputed prior to the online ROM verification simulation following Eq. 13:

$$
\begin{aligned}
\frac{\mathrm{d} \mathbf{T}_{r}(t)}{\mathrm{d} t}= & \left(\mathbf{U}_{r}^{T} \mathbf{C}_{A}\right) \mathbf{P}_{A}^{T} \mathbf{A} \mathbf{U}_{r} \mathbf{T}_{r}(t) \\
& +\left(\mathbf{U}_{r}^{T} \mathbf{C}_{R}\right) \mathbf{P}_{R}^{T} \mathbf{R}\left(\mathbf{U}_{r} \mathbf{T}_{r}(t)\right)^{* 4}+\mathbf{D}_{r}+\left(\mathbf{U}_{r}^{T} \mathbf{C}_{B}\right) \mathbf{P}_{B}^{T} \mathbf{B} \mathbf{u},
\end{aligned}
$$

where $\mathbf{C}$ in each term on the RHS of Eq. 23 can be written as:

$\mathbf{C}_{i}=\mathbf{U}_{i}\left(\mathbf{P}_{i}^{T} \mathbf{U}_{i}\right)^{-1}$,

where subscript $i$ can be $A, R$, and $B$ to represent the quantities for the nonlinear term, $\mathbf{A T}(t), \mathbf{R T}(t)^{* 4}$ and $\mathbf{B u}$, respectively. In Eq. 23, $\mathbf{P}_{A}^{T} \mathbf{A}, \mathbf{P}_{R}^{T} \mathbf{R}$, and $\mathbf{P}_{B}^{T} \mathbf{B}$ clearly show that only $m_{A}, m_{R}$, and $m_{B}$ rows of $\mathbf{A}, \mathbf{R}$, and $\mathbf{B}$ matrices corresponding to the DEIM cells need to be assembled, and hence, reducing the computing cost. In fact, since $\mathbf{R}$ matrix is diagonal and subscript $*$ denotes element-wise operation, $\mathbf{P}_{R}^{T} \mathbf{R}\left(\mathbf{U}_{r} \mathbf{T}_{r}(t)\right)^{* 4}$ in the $2^{\text {nd }}$ nonlinear term associated with external radiation can be further simplified by $\mathbf{P}_{R}^{T} \mathbf{R} \mathbf{P}_{R}\left(\mathbf{P}_{R}^{T} \mathbf{U}_{r} \mathbf{T}_{r}(t)\right)^{* 4}$, which signifies that only $m_{R}$ columns of $\mathbf{R}$ and $m_{R}$ rows of $\mathbf{U}_{r}$ at the DEIM cells will be used in matrix and vector multiplication to further improve efficiency. Equation 23 eventually can be written in a more concise form used for verification simulation:

$$
\begin{aligned}
\frac{\mathrm{d} \mathbf{T}_{r}(t)}{\mathrm{d} t}= & \mathbf{Q}_{A} \mathbf{A}_{P} \mathbf{U}_{r} \mathbf{T}_{r}(t)+\mathbf{Q}_{R} \mathbf{R}_{P}\left(\hat{\mathbf{U}}_{r} \mathbf{T}_{r}(t)\right)^{* 4}+\mathbf{D}_{r} \\
& +\mathbf{Q}_{B} \mathbf{B}_{P} \mathbf{U}_{r}
\end{aligned}
$$

where the matrices:

$\mathbf{Q}_{A}=\mathbf{U}_{r}^{T} \mathbf{C}_{A}$,

$\mathbf{Q}_{R}=\mathbf{U}_{r}^{T} \mathbf{C}_{R}$,

$\mathbf{Q}_{B}=\mathbf{U}_{r}^{T} \mathbf{C}_{B}$

combine the subspace projection onto $\mathbf{U}_{r}$ with DEIM interpolation terms in Eq. 23 and can be precomputed. 


$$
\begin{aligned}
& \mathbf{A}_{P}=\mathbf{P}_{A}^{T} \mathbf{A}, \\
& \mathbf{B}_{P}=\mathbf{P}_{B}^{T} \mathbf{B}
\end{aligned}
$$

indicate that only rows at DEIM cells will be assembled for $\mathbf{A}$ and $\mathbf{B}$ matrix.

$\mathbf{R}_{P}=\mathbf{P}_{R}^{T} \mathbf{R} \mathbf{P}_{R}$

means only rows and columns of $\mathbf{R}$ matrix at DEIM cells will beassembled because it is a diagonal matrix, and accordingly $\hat{\mathbf{U}}_{r}$ denotes the rows of $\mathbf{U}_{r}$ selected at DEIM cells.

The temporal differential term of the ODE set of the FOM and ROM is discretized by the second-order, implicit Crank-Nicolson method. The resultant linear equation set is solved using Matlab's built-in function " $\backslash$ " (backslash), which examines on the matrix properties and determines an appropriate solution technique to minimize computation time.

\section{Numerical results and discussions}

In this paper, two numerical case studies based on the aforementioned nonlinear heat transfer model are performed to validate the feasibility of hybrid snapshot simulation for ROM-DEIM construction. The first case study is an annular cone model with two layers of equal thickness, including an outer layer made of stainless and an inner layer made of mineral wool. This model is discretized into 9408 mesh cells, and the geometry and the grids are shown in Fig. 6a. The adiabatic boundary condition is applied at the bottom, the top, the inner wall, and a quarter of the outer lateral wall. A time-varying heat flux is prescribed at the rest of the outer lateral walls (the rest three quarters), and the added heat could be transported to the interior of the cone through conduction or dissipated from the outer boundaries to the environment by thermal radiation. The profiles of the time-varying heat fluxes in both the snapshot simulation and the online verification simulation are depicted in Fig. 6 b.

One part of the model nonlinearity is introduced through the temperature-dependent thermal conductivity of both materials as $k=11.13+1.27 \times 10^{-2} \times T$ for stainless steel and $k=0.03+0.2438 \times\left(\frac{T}{1000}\right)^{3}$ for mineral wool. In order to confirm its importance, another analysis, which takes constant thermal conductivity at room temperature $(300 \mathrm{~K})$, i.e., $k=15 \mathrm{~W} / \mathrm{mK}$ and $0.0366 \mathrm{~W} / \mathrm{mK}$, is also performed and used as a benchmark for comparison. Other physical properties are the density is $\rho=8030 \mathrm{~kg} / \mathrm{m}^{3}$ for stainless steel and $\rho=165 \mathrm{~kg} / \mathrm{m}^{3}$ for mineral wool; the specific heat is $c_{p}=510 \mathrm{~J} / \mathrm{kg} \mathrm{K}$ for stainless steel and $c_{p}=840 \mathrm{~J} / \mathrm{kg} \mathrm{K}$ for mineral wool. In addition, we also assume the radiative emissivity at the outer wall is 1 .

The second case study considers the heat transfer through a fin array made of stainless steel, which includes thermal conduction within the fin array and the radiation from the fin surface to the environment. The geometry and computational grids of the model are presented in Fig. $7 \mathrm{a}$, and the total number of cells is 38,400 . The lateral faces of the substrate are given the adiabatic boundary condition. The bottom of the substrate is assigned to a boundary condition of time-varying heat flux, whose profiles for the snapshot simulation and the online verification simulation are shown in Fig. 7b. For the rest surfaces, the radiation boundary condition is prescribed with the far field environmental temperature set to $300 \mathrm{~K}$. Again two analyses using constant thermal conductivity $k=15 \mathrm{~W} / \mathrm{mK}$ and temperature-dependent thermal conductivity, which is $k=11.13+1.27 \times 10^{-2} \times T$ for stainless steel and $k=0.03+0.2438 \times\left(\frac{T}{1000}\right)^{3}$ for mineral wool, are performed to compare both results and confirm the significance of the model nonlinearity. We also use the radiative emissivity of 1 at the surface of the fin array.

Following the procedure presented above, the hybrid snapshot simulation is undertaken, and it alternates between FOM and ROM according to the criteria. iSVD is
Fig. 6 The numerical model of the multi-layer annular cone: a geometry and computational grids; and $\mathbf{b}$ the heat flux profile prescribed at the three quarters of the outer wall of the model and used in the snapshot simulation (in pink) and the online verification simulation (in green)

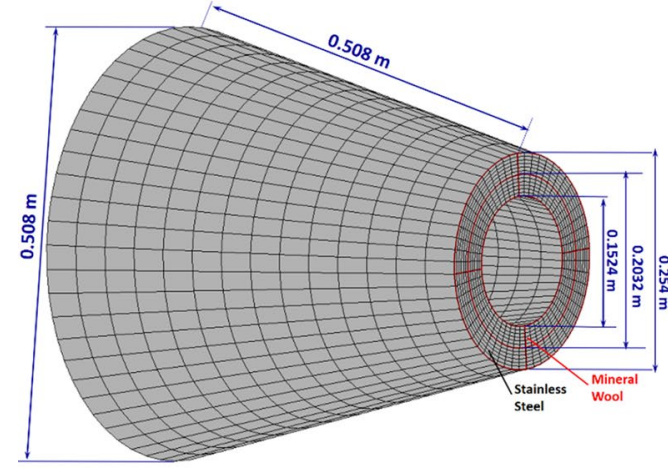

(a)

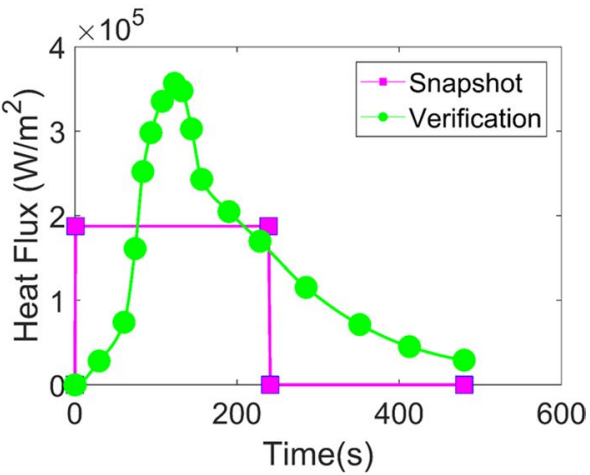

(b) 


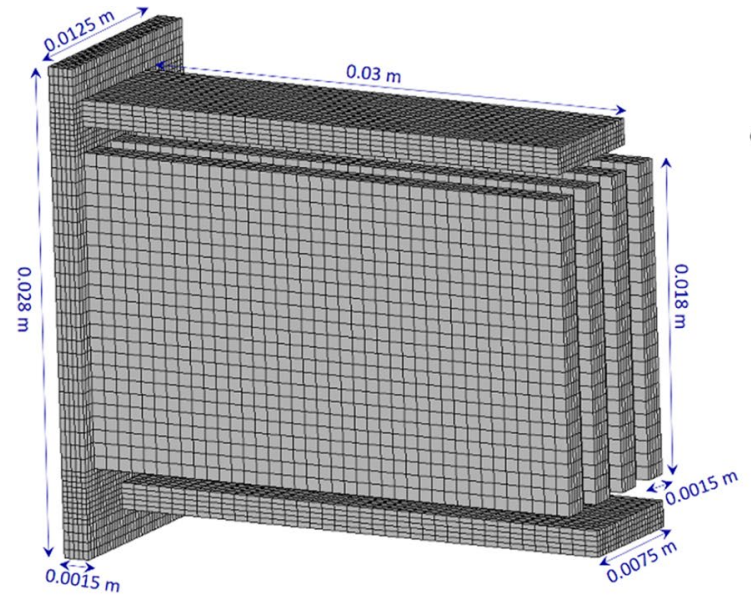

(a)

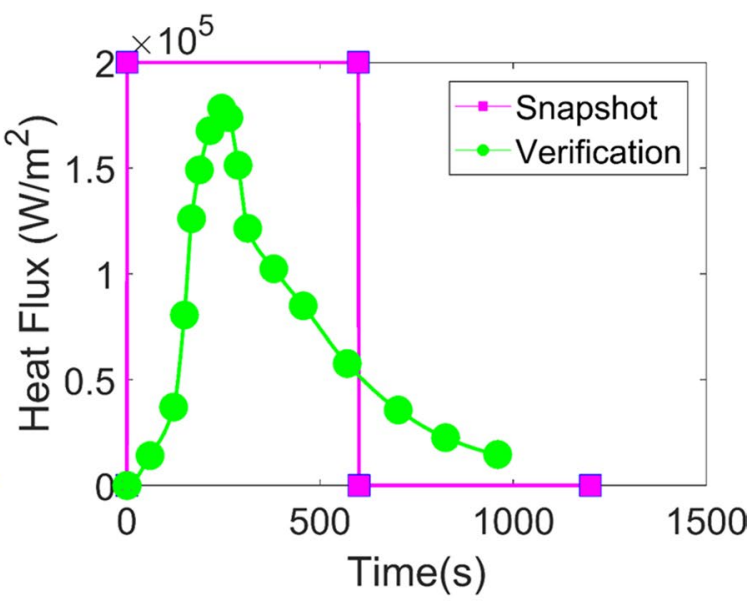

(b)

Fig. 7 The model of extended fin arrays: a geometry and grids; and $\mathbf{b}$ the heat flux profile prescribed at the bottom of the substrate of the computational model and used both in the snapshot simulation (in pink) and the verification simulation (in green)

used to update the POD modes of the temperature solution at the end of each FOM interval. At the end of snapshot simulation, the snapshot data of all the nonlinear terms are combined, from which the POD modes, and indices of DEIM interpolation points (i.e., DEIM cells) and their adjacent cells are also obtained. Then, ROM-DEIM is built, and the online verification simulation is carried out. Three ROMs are compared, including ROM obtained from FOM snapshot simulation, ROM from hybrid snapshot simulation without DEIM, and ROM with DEIM (i.e., ROM-DEIM).

Since various simulation schemes and models are used in the snapshot simulation and the verification simulation, several abbreviations are defined first to facilitate result discussion below. For snapshot simulation, SS-Hybrid is defined to represent the hybrid simulation that alternates between FOM and ROM simulation to generate snapshot data. The ROM obtained in this way without DEIM is termed HROM. Alternatively, at the end of SS-Hybrid, the solution during the ROM interval can be used to reconstruct the data in the full domain, which is then combined with FOM data to form the mixed snapshot data of the nonlinear terms. The POD modes and the DEIM interpolation indices of the nonlinear terms then can be computed. The ROM constructed by this process is named HROMDEIM, which is the main contribution of the present effort. The traditional snapshot simulation exclusively using FOM is named SS-FOM, which is also used as the benchmark for comparison with other ROMs. In SS-FOM, the POD modes will be extracted only at the end of the snapshot simulation, and the ROM constructed is termed FROM.

In the online verification simulation, the three ROMs built by different methods above, including HROM, HROM-DEIM, and FROM, will be compared in terms of accuracy and computational speed. Our investigation indicates that the assembly of the inter-cell transport-related term, i.e., $\mathbf{A T}(t)$ is most time consuming as it involves all the interior cells rather than only the boundary cells, and the number of the DEIM cells (interpolation points) notably affects the accuracy of HROM-DEIM. Therefore, in this paper, $m_{A}$ is varied to examine its influence on accuracy and computational time, while keeping the DEIM numbers in the cell-wise terms (i.e., $m_{R}$ and $m_{B}$ ) fixed.

In order to examine the errors quantitatively, two error metrics widely used for FOM and ROM comparison, including the absolute error $\mathbf{E r r}_{\mathrm{abs}}$ and the mean square relative difference(MSRD) $\mathbf{E r r}_{\text {msrd }}[5,48]$, are defined:

$\operatorname{Err}_{\mathrm{abs}}(t)=\left|\mathbf{U}_{r} \mathbf{T}_{r}(t)-\mathbf{T}(t)\right|$,

$\mathbf{E r r}_{\mathrm{msrd}}(t)=\sqrt{\frac{\sum_{j=1}^{n}\left(\frac{\left(\mathbf{U}_{r} \mathbf{T}_{r}(t)\right)_{j}-\mathbf{T}_{j}(t)}{\mathbf{T}_{j}(t)}\right)^{2}}{n}} \times 100 \%$,

where subscript $j$ denotes the $j$ th row of the reconstructed temperature solution $\mathbf{U}_{r} \mathbf{T}_{r}(t)$ corresponding to the $j$ th cell; $\mathbf{T}_{r}$ is the ROM/ROM-DEIM solutions of the temperature in the $j$ th cell; and $\mathbf{T}_{j}$ is the FOM solutions at the $j$ th cell. It should be noted that both of these errors $\mathbf{E r r}_{\text {abs }}(t)$ and $\mathbf{E r r}_{\text {msrd }}(t)$ vary with time. The common parameters in the numerical model and computation in both case studies are listed as follows: The number of basic and extended POD modes is $r_{d}=20$ and $r_{e}=4$, respectively; the FOM/ ROM switch tolerance parameters are $\epsilon_{a}=1 e^{-5}, \epsilon_{b}=1 e^{-6}$, and $\epsilon=1 e^{-4}$. The models presented in this paper are programmed and simulated in Matlab 2017b. All of the 
numerical tests are performed on a PC equipped with an Intel i7-7700 CPU with a clock speed of $3.60 \mathrm{GHz}$ and the RAM of $32 \mathrm{~GB}$. The operating system is Windows 10 Enterprise 2016LTSB.

\subsection{Case study 1}

For the annular cone model, the total time span of the snapshot simulation is $480 \mathrm{~s}$, which is divided into 12 time intervals, corresponding $\Delta t=40 \mathrm{~s}$ for each interval. The time span for online verification simulation is also $480 \mathrm{~s}$, in which various ROMs will be simulated and their results will be compared with FOMs. In both the snapshot and online verification simulation, the time step is $\delta t=0.25 \mathrm{~s}$.

Figure 8 shows the numerical results obtained in the snapshot simulation using FOM only (i.e., SS-FOM) and the absolute error of the snapshot data computed by the hybrid FOM/ROM (i.e., SS-Hybrid) relative to SS-FOM. Note that SS-hybrid data are not shown here, because SS-FOM and SS-Hybrid snapshot data are nearly the same with very small errors that are almost visually indistinguishable (maximum $\mathbf{E r r}_{\text {abs }} \leq 10 \mathrm{~K}$ with respect to the peak temperature $\sim 800 \mathrm{~K}$ ). This also confirms the accuracy of SS-Hybrid to generate snapshot data. As shown in Table 1, among 12 intervals, there are four intervals and eight intervals, respectively, simulated by FOM and ROM. That is, the first, fifth, eighth and tenth intervals are the FOM interval, and the rest are the ROM interval. Table 1 also lists the computational time breakdown of each interval during the entire snapshot simulation, including the FOM simulation (four intervals), the iSVD after each FOM interval ( 3 iSVD and 1 SVD), and the ROM simulation (eight intervals). Note that following the initial FOM interval the
Table 1 The breakdown of the computational time for the hybrid snapshot simulation for the annular cone model (Unit:s)

\begin{tabular}{llll}
\hline Time section & FOM & iSVD & ROM \\
\hline First & 36.767 & 0.058 & $\ldots$ \\
Second & $\ldots$ & $\ldots$ & 4.476 \\
Third & $\ldots$ & $\ldots$ & 4.641 \\
Fourth & $\ldots$ & $\ldots$ & 4.553 \\
Fifth & 19.385 & 0.229 & $\ldots$ \\
Sixth & $\ldots$ & $\ldots$ & 4.431 \\
Seventh & $\ldots$ & $\ldots$ & 4.770 \\
Eighth & 18.981 & 0.168 & $\ldots$ \\
Ninth & $\ldots$ & $\ldots$ & 4.522 \\
Tenth & 20.061 & 0.173 & $\ldots$ \\
Eleventh & $\ldots$ & $\ldots$ & 4.863 \\
Twelfth & $\ldots$ & $\ldots$ & 4.681 \\
Total & $\mathbf{9 5 . 1 9 4}$ & $\mathbf{0 . 6 2 8}$ & $\mathbf{3 6 . 9 3 7}$ \\
\hline
\end{tabular}

SVD is applied rather than iSVD to construct the initial POD modes. It also indicates that with the hybrid method, eight out of 12 intervals are simulated by ROM in place of FOM to generate the snapshot data without appreciably compromising the data accuracy and quality. The computational time of ROM is greatly less than FOM, and hence, accelerating the snapshot simulation. Quantitatively, the total simulation time of SS-Hybrid and SS-FOM is, respectively, $132.759 \mathrm{~s}$ and $264.564 \mathrm{~s}$, yielding 50\% speedup.

Next, several ROMs constructed by different methods are compared in the online verification simulation, including FROM by SS-FOM, HROM by SS-Hybrid, and HROMDEIM by SS-Hybrid. The solution of the FROM and HROM is shown in Fig. 9a-1 and b-1, and their comparisons with

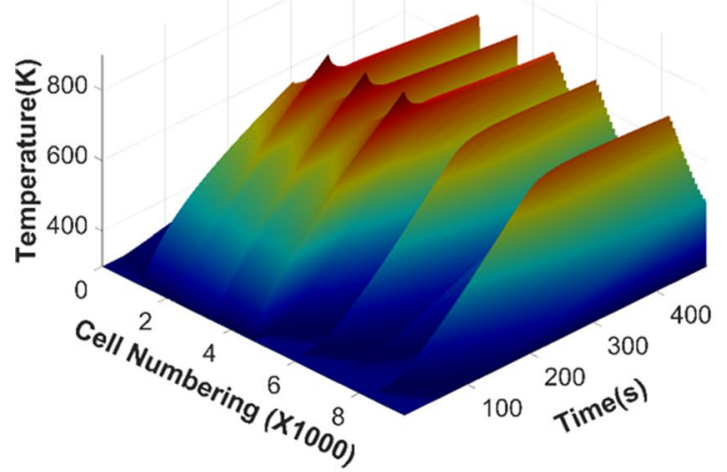

(a)

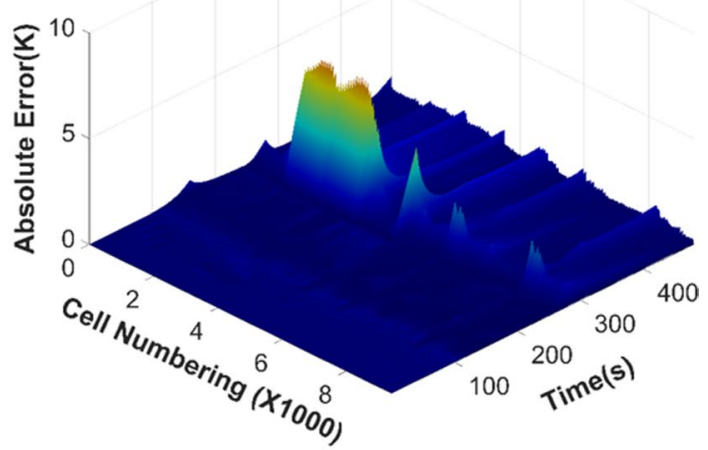

(b)

Fig. 8 The comparison between SS-FOM and SS-Hybrid in the annular cone model: $\mathbf{a}$ the snapshot data produced by SS-FOM and $\mathbf{b}$ the absolute error between SS-FOM and SS-Hybrid 


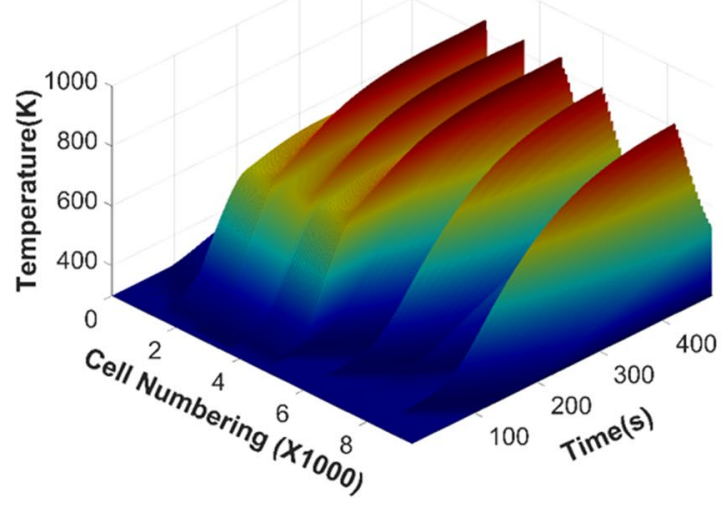

(a-1)

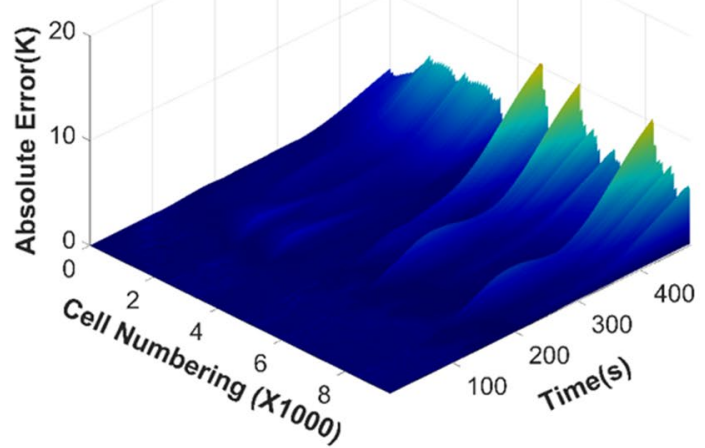

$(\mathrm{a}-2)$

Fig. 9 The comparison of FROM and HROM with FOM for the annular cone model: the temperature solution of all the computational cells throughout the verification simulation obtained by a-1 FROM

the FOM solution, i.e., $\mathbf{E r r}_{\text {abs }}$ are presented in Fig. 9a-2 and $b-2$. It is clear that solutions of FROM and HROM are nearly the same with negligible difference, which confirms the accuracy of ROM and POD modes constructed using the hybrid snapshot simulation. Figure $9 a-2$ and b-2 show that absolute errors $\mathbf{E r r}_{\text {abs }}$ of both FROM and HROM increase with time and reach the highest values of $12 \mathrm{~K}$ at the end of the verification simulation, when the temperature of the whole model also approaches the peak. The value of $\mathbf{E r r}_{\text {abs }}$ of HROM is slightly higher than that of FROM, because less high-fidelity FOM data are generated in SS-Hybrid.

Next HROM-DEIM is constructed for the verification simulation in order to reduce the computational cost associated with the nonlinear term calculation in the reduced domain. By keeping constant the number of the DEIM cells (interpolation points) for all the cell-wise terms $\left(m_{R}=100\right.$

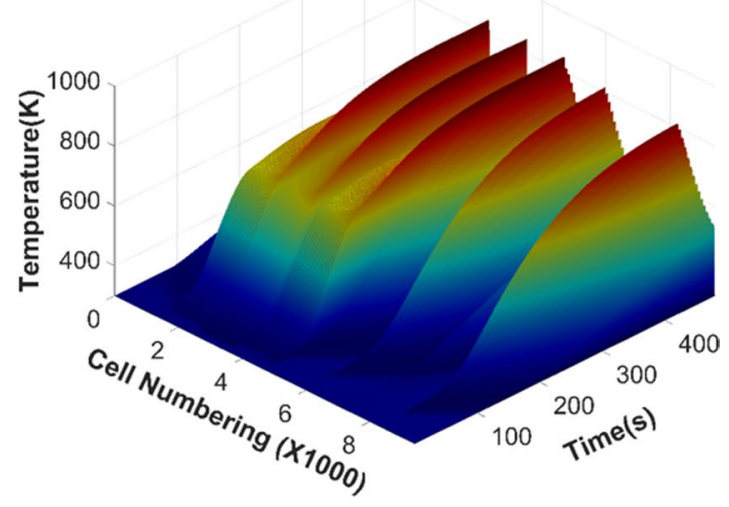

(b-1)

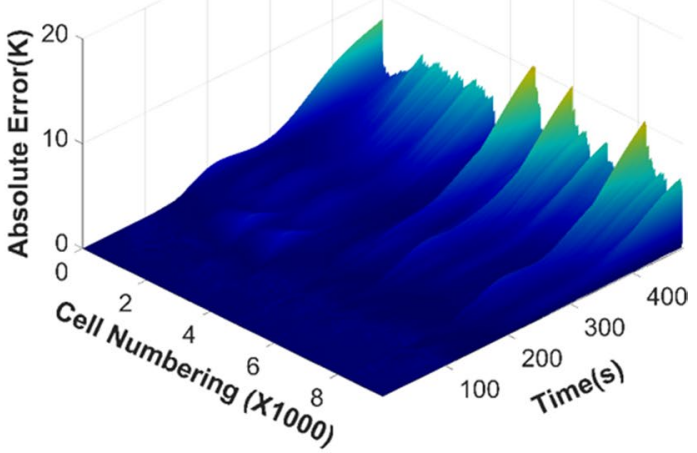

(b-2)

and b-1 HROM; and the absolute error Err $_{\mathrm{abs}}$ of a-2 FROM and b-2 HROM relative to FOM

and $m_{B}=100$ ), we investigate the effect of the DEIM cell number of the inter-cell transport term on accuracy and speed. The rationale for us to choose a large number of DEIM cells for the cell-wise nonlinear terms is to ensure that they can be approximated with higher accuracy and the effect of DEIM interpolation on them can be minimized. Thus, the analysis can be concentrated on the intercell transport nonlinear term. Figure 10 illustrates the temperature solution of all computational cells throughout the verification simulation produced by the three HROMDEIMs with the different number of DEIM cells for the inter-cell nonlinear term $\left(m_{A}=40,60\right.$ and 80$)$ and HROM without DEIM. It is observed that the numerical results for all of them are almost the same in the entire spatiotemporal domain. The initial temperature values are equal to the environmental one $(300 \mathrm{~K})$. The temperature rises quickly between 100 and $300 \mathrm{~s}$, and eventually, it reaches 


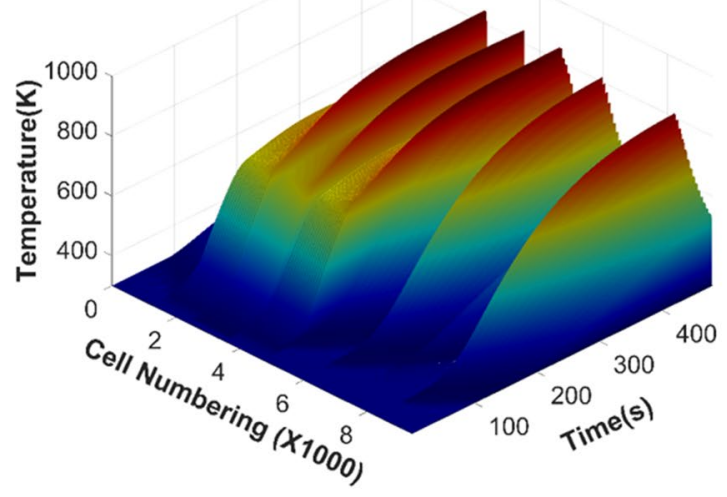

(a)

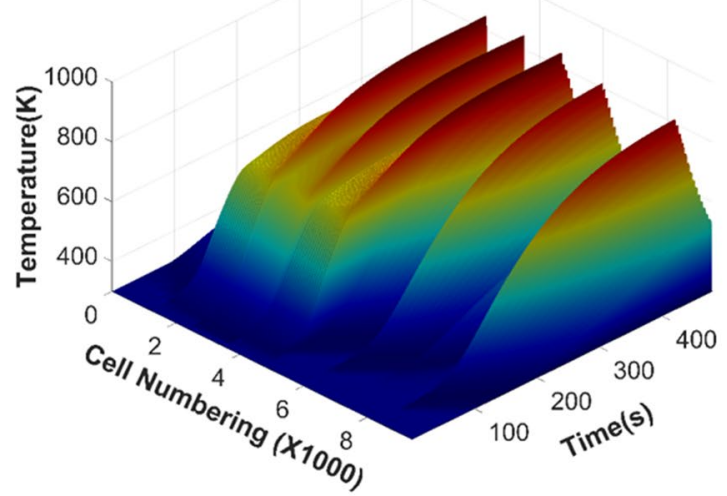

(c)

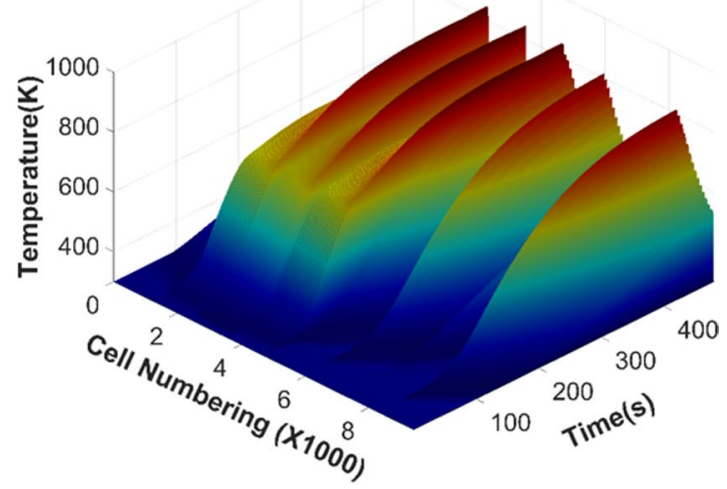

(b)

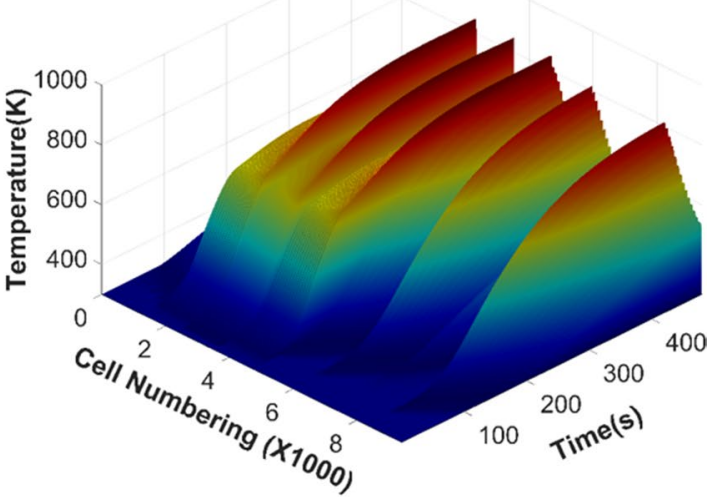

(d)

Fig. 10 The temperature solutions obtained by HROM-DEIM with the different number of DEIM cells in the annular cone model: a DEIM 40 $\left(m_{A}=40\right), \mathbf{b}$ DEIM $60\left(m_{A}=60\right), \mathbf{c}$ DEIM $80\left(m_{A}=80\right)$ and d non-DEIM

the highest value about $850 \mathrm{~K}$ at the end of the simulation. Figure 11 illustrates the absolute errors $\mathbf{E r r}_{\text {abs }}$ of the numerical results of the HROM-DEIM in Fig. 10 relative to the FOM solution. It clearly shows that $\mathbf{E r r}_{\text {abs }}$ increases with time for all the computational cells, and there seems a trend that $\mathbf{E r r}_{\text {abs }}$ is larger when a smaller number of DEIM cells are used. The maximum value of $\mathbf{E r r}_{\text {abs }}$ produced by HROM-DEIM with $m_{A}=40$ is about $15 \mathrm{~K}$, while it is less than $10 \mathrm{~K}$ with $m_{A}=80$. In addition, it is also observed that the values of $\mathbf{E r r}_{\text {abs }}$ versus time for HROM (Fig. 11d) are slightly lower than that of HROM-DEIM (Fig. 11a-C), which verifies that HROM without DEIM interpolation (or approximation) is more accurate than HROM-DEIM. However, with DEIM, the computational complexity and cost could be reduced significantly, leading to salient simulation acceleration which is as follows.

The values of the mean square root difference error(MSRD) $\mathbf{E r r}_{\text {msrd }}$ of all the ROM solutions in Fig. 11 relative to FOM are shown in Fig. 12. A trend similar to Fig. 11 is observed. That is, $\mathbf{E r r}_{\text {msrd }}$ seems to decrease as a result of the increasing number of DEIM cells, and $\mathbf{E r} \mathbf{r}_{\text {msrd }}$ of HROM without DEIM is the lowest in the comparison. Even with only 40 DEIM cells (i.e., $m_{A}=40$ ), $\mathbf{E r r}_{\text {msrd }}$ is still less than $0.8 \%$. This indicates that DEIM interpolation of the nonlinear inter-cell transport (such as temperaturedependent thermal conduction in this article) is an effective means to improve nonlinear ROM simulation speed while preserving the accuracy. It should be noted that DEIM selects the cells or interpolation points based on the largest magnitude of the residual of reconstructing the next basis vector in POD modes [15, 17]; therefore, which is not necessarily equivalent to ROM accuracy.

Table 2 lists the computational time of FOM, HROM, and HROM-DEIM in the verification simulation. It is clearly seen that ROMs could accelerate the simulation greatly, and the computational time of HROM is only about $18.6 \%$ 


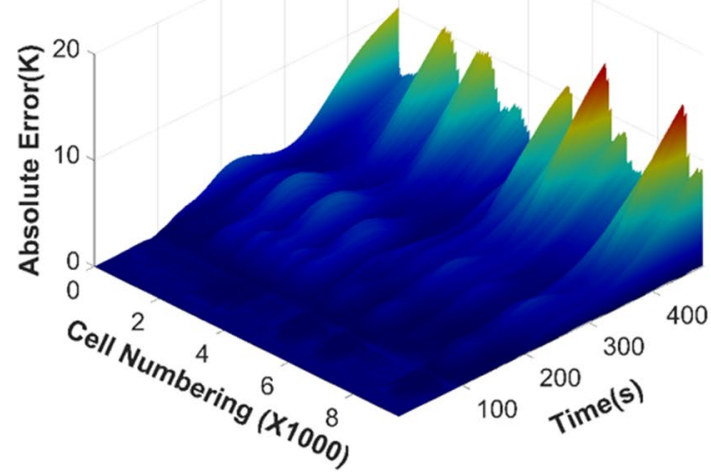

(a)

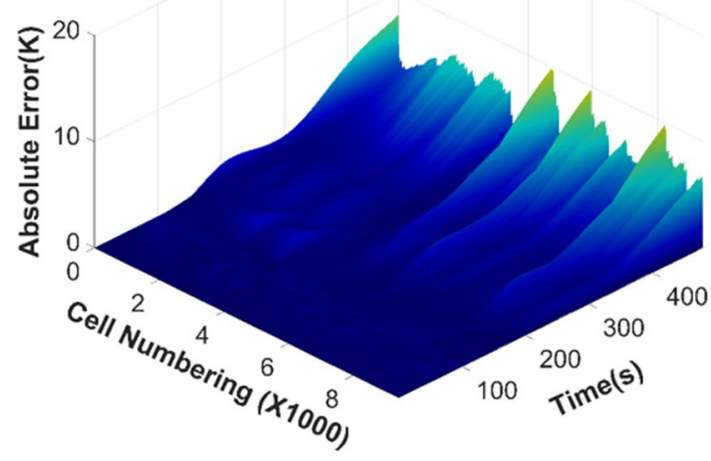

(c)

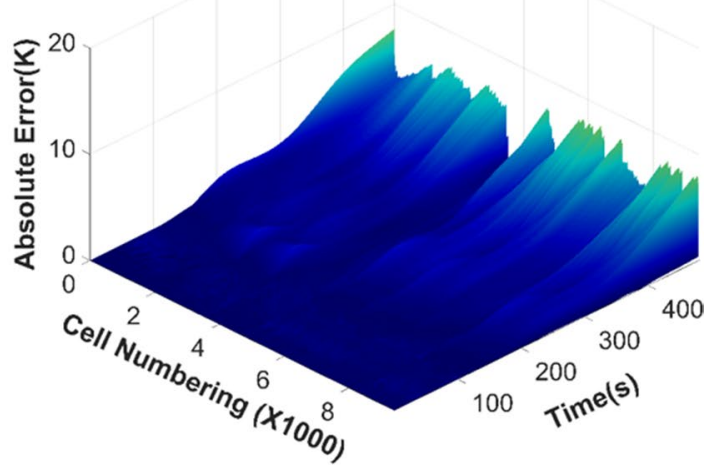

(b)

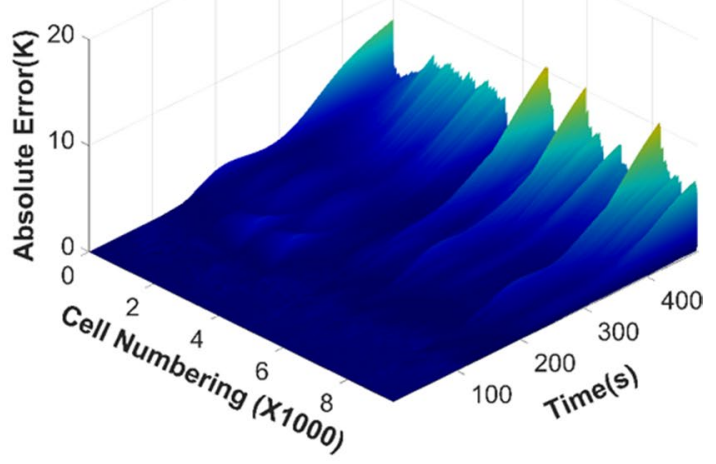

(d)

Fig. 11 The absolute error of the temperature solution obtained by HROM-DEIM with the different number of DEIM cells in the annular cone model relative to the FOM solution: a DEIM $40\left(m_{A}=40\right), \mathbf{b}$ DEIM $60\left(m_{A}=60\right), \mathbf{c}$ DEIM $80\left(m_{A}=80\right)$ and d non-DEIM

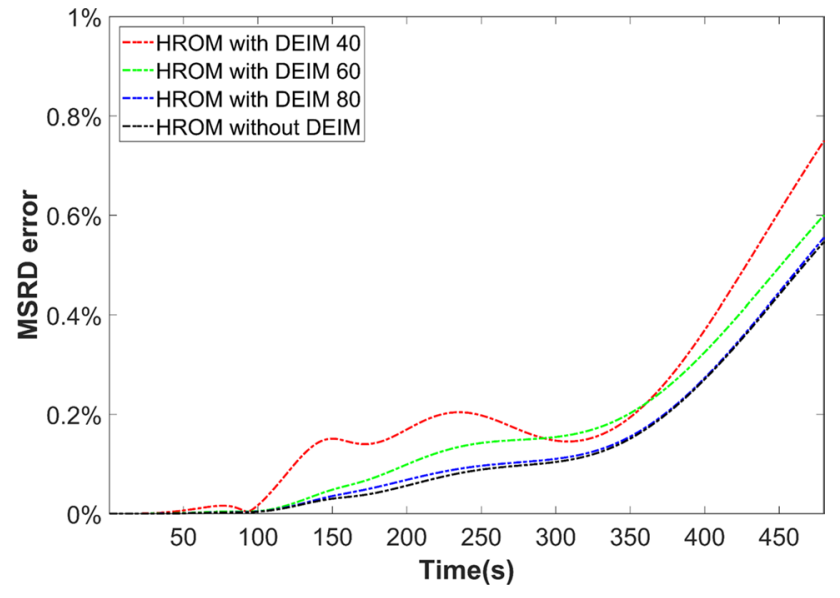

Fig. 12 The MSRD errors versus time for HROM-DEIM with the different number of DEIM cells and HROM without DEIM in the verification simulation in the annular cone model
Table 2 The comparison of computational time in FOM, HROM, and HROM-DEIM in the annular cone model (Unit:s)

\begin{tabular}{llllll}
\hline Model type & FOM & HROM & \multicolumn{2}{l}{ HROM-DEIM } \\
\cline { 3 - 6 } & & & $m_{A}=40$ & $m_{A}=60$ & $m_{A}=80$ \\
\hline Time & 293.517 & 54.702 & 15.065 & 15.117 & 15.459 \\
\hline
\end{tabular}

of FOM. However, HROM-DEIM is able to achieve an even higher acceleration than HROM due to the reduced cost of assembling the nonlinear terms. The ratio of the computational time of HROM-DEIM to HROM is $27.5 \%$ and to FOM is only $5.13-5.27 \%$, the latter of which indicates that the time usage by HROM-DEIM is almost negligible compared with the traditional method. Most importantly, with the hybrid simulation method, the snapshot generation used to construct the HROM-DEIM can be reduced by almost 
half (see Table 1). In addition, despite various $m_{A}$ used (40, 60 , and 80 ) in HROM-DEIMs, they can be simulated almost with the same time $\sim 15 \mathrm{~s}$, because all $m_{A} \mathrm{~s}$ are quite small compared with the dimension of FOM.

For the annular cone model, the difference caused by the temperature-dependent thermal conductivity relative to the constant thermal conductivity is also briefly examined in order to demonstrate that the inter-cell transportinduced nonlinearity in this paper plays an important role in accurate modeling. The values of temperaturedependent conductivity and the constant conductivity in constituent materials are given above. Figure 13 shows the temperature solution of FOM computed using the constant conductivity, and its difference ( $\mathbf{E r r}_{\text {abs }}$ ) relative to the solution using temperature-dependent conductivity. For the sake of paper conciseness, the FOM solutions are not shown as they are almost the same as those in Figs. 9 and 10. It clearly shows that the difference $\left(\mathbf{E r r}_{\text {abs }}\right)$ is larger as the temperature increases with time, and the maximum could reach nearly $200 \mathrm{~K}$ in the sub-domain containing the material of low thermal conductivity that is more sensitive to the temperature. Therefore, it is confirmed that the temperature dependent, inter-cell transport phenomenon gives rise to notable model nonlinearity, and the nonlinear term updating and assembly during each iteration and each time step is necessary.

\subsection{Case study 2}

In the second case study of the extended fin array model, the total time span of the snapshot simulation is $1200 \mathrm{~s}$

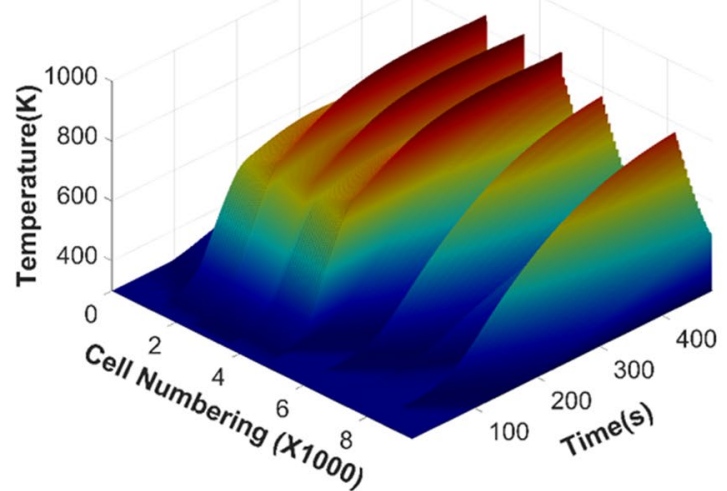

(a) and divided into 12 time intervals for the hybrid FOM/ROM simulation, yielding $\Delta t=100 \mathrm{~s}$ for each time interval. The time span for the entire online verification simulation is $960 \mathrm{~s}$. The time step for both the snapshot and online verification simulation is $\delta t=0.5 \mathrm{~s}$.

Figure 14a shows the numerical results of the temperature solution generated using SS-FOM in the snapshot simulation, and Fig. $14 \mathrm{~b}$ portrays the absolute error $\mathbf{E r r}_{\text {abs }}$ of the snapshot data between SS-FOM and SS-Hybrid for all the computational cells throughout the entire time span. Again in order to avoid presenting redundant data, the temperature solution of SS-Hybrid is not shown. We can see that the maximum value of $\mathbf{E r r}_{\text {abs }}$ is less than $8 \mathrm{~K}$ and is negligible considering the largest magnitude of the snapshot data (up to $1100 \mathrm{~K}$ ), which again substantiates the robustness and accuracy of the hybrid snapshot simulation.

The breakdown of the computational time using SSHybrid in the snapshot simulation is listed in Table. 3. It can be seen that among the 12 time intervals, there are only two intervals simulated by FOM (the first and the eighth) and the rest ten intervals all by ROM. Except for the first FOM interval that uses SVD to construct the initial POD modes, iSVD is only applied once following the eighth interval in the whole snapshot simulation. The table also clearly shows that the snapshot simulation switches to ten ROM intervals based on the FOM/ROM criteria, and the total computational time (489.686 s) is greatly lowered compared with the traditional FOM-based snapshot simulation (929.748 s), i.e., $47 \%$ reduction.

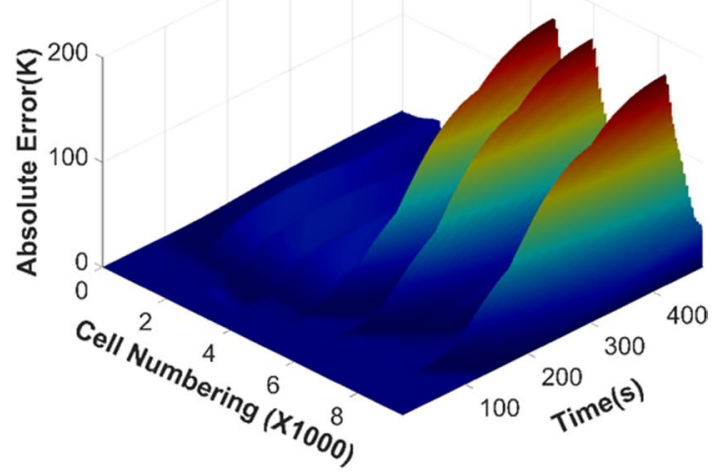

(b)

ductivity; and $\mathbf{b}$ the absolute error between the solution in $\mathbf{a}$ and the solution obtained using temperature-dependent thermal conductivity
Fig. 13 Effect of the temperature-dependent thermal conductivity on model nonlinearity: a the temperature solution obtained by FOM in the annular cone model using the constant thermal con-

\section{SN Applied Sciences}




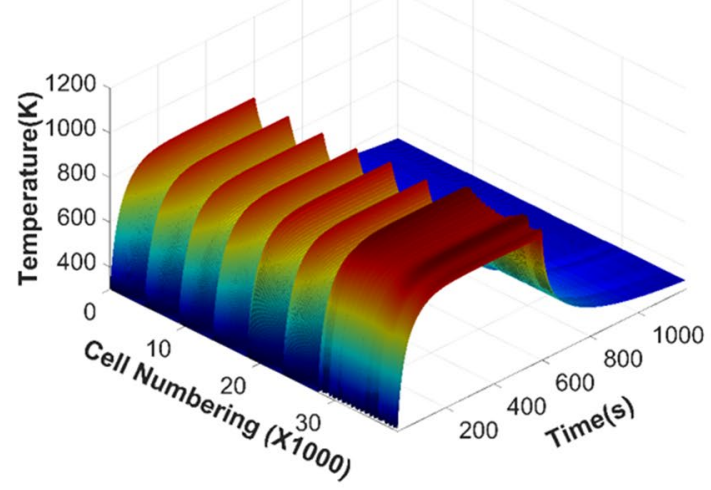

(a)

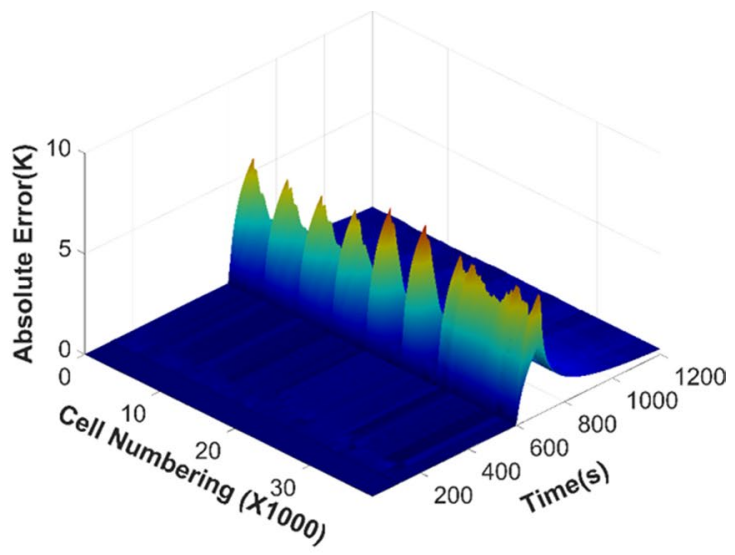

(b)

Fig. 14 The comparison between SS-FOM and SS-Hybrid in the extended fin array model: $\mathbf{a}$ the snapshot data produced by SS-FOM and $\mathbf{b}$ the absolute error between SS-FOM and SS-Hybrid

Table 3 The breakdown of the computational time for the hybrid snapshot simulation for the extended fins model (Unit:s)

\begin{tabular}{llll}
\hline Time section & FOM & iSVD & ROM \\
\hline First & 123.085 & 0.362 & $\ldots$ \\
Second & $\ldots$ & $\ldots$ & 29.270 \\
Third & $\ldots$ & $\ldots$ & 28.072 \\
Fourth & $\ldots$ & $\ldots$ & 28.146 \\
Fifth & $\ldots$ & $\ldots$ & 28.051 \\
Sixth & $\ldots$ & $\ldots$ & 28.003 \\
Seventh & $\ldots$ & $\ldots$ & 41.118 \\
Eighth & 72.070 & 1.031 & $\ldots$ \\
Ninth & $\ldots$ & $\ldots$ & 27.902 \\
Tenth & $\ldots$ & $\ldots$ & 27.534 \\
Eleventh & $\ldots$ & $\ldots$ & 27.226 \\
Twelfth & $\ldots$ & $\ldots$ & 27.816 \\
Total & $\mathbf{1 9 5 . 1 5 5}$ & $\mathbf{1 . 3 9 3}$ & $\mathbf{2 9 3 . 1 3 8}$ \\
\hline
\end{tabular}

Next, we will compare the performance of all the ROMs in the verification simulation. Figure $15 a-1$ and b-1 illustrates the numerical results of the temperature solution obtained by FROM and HROM, respectively. Again, both exhibit excellent agreement with a very minor difference. Their absolute errors $\mathbf{E r r}_{\text {abs }}$ relative to the FOM solution are shown in Fig. 15a-2 and b-2, and the values of both Err $_{\text {abs }}$ are extremely low $(\leq 0.5 \mathrm{~K})$ and indeed negligible compared with the absolute values of the temperature solution, although $\mathbf{E r r}_{\text {abs }}$ of HROM is somewhat higher than FROM. The observations also indicate that the POD modes and ROM constructed by the hybrid snapshot method are accurate to be utilized in the online verification simulation.

The temperature solutions in the online verification simulation obtained by the three HROM-DEIMs with the different number of DEIM cells $\left(m_{A}=50,75\right.$ and 100) and HROM without DEIM are illustrated in Fig. 16. Similar to the previous case study, all the numerical results are almost identical with a tiny difference. The temperature rises up rapidly in the first $300 \mathrm{~s}$ until arriving the peak value $(\sim 1000 \mathrm{~K})$ and then gradually declines to about $450 \mathrm{~K}$ at the end of the simulation as a result of the decreasing heat flux applied. Their absolute errors $\mathbf{E r r}_{\text {abs }}$ relative to the FOM solution are shown in Fig. 17, and the profiles of $\mathbf{E r r}_{\text {abs }}$ in all sub-figures exhibit the similar trend: $\mathbf{E r r}_{\mathrm{abs}}$ grows quickly from 0 to $200 \mathrm{~s}$, decreases a little from 200 to $300 \mathrm{~s}$, and then mostly maintains at approximately the same level from $300 \mathrm{~s}$ till the end. Figure 17 also seems to reveal the correlation between the DEIM cell number and the magnitude of $\mathbf{E r r}_{\text {abs, }}$ that is, $\mathbf{E r r}_{\text {abs }}$ decreases as the number of DEIM cells for the inter-cell transport term increases from $m_{A}=50$ to $m_{A}=100$. It is also noticed that Err $_{\text {abs }}$ of HROM-DEIM (Fig. 17c) is slightly higher than 


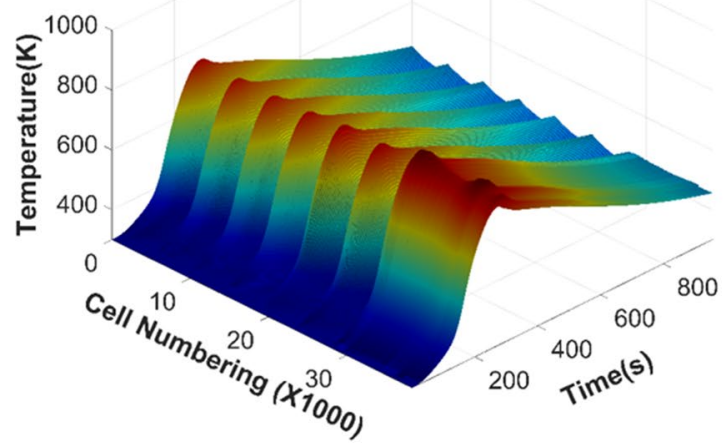

$(\mathrm{a}-1)$

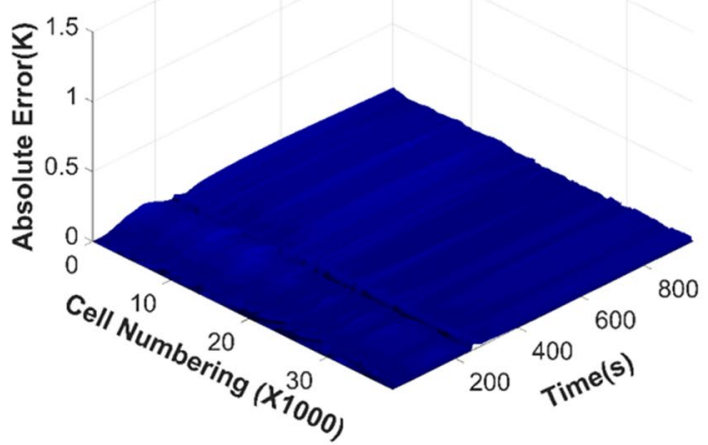

$(\mathrm{a}-2)$

Fig. 15 The comparison of FROM and HROM with FOM for the extended fin array model: the temperature solution of all the computational cells throughout the verification simulation obtained by

that of HROM (Fig. 17d) because no DEIM interpolation or approximation is used in the latter.

Figure 18 shows the MSRD error $\mathbf{E r r}_{\text {msrd }}$ of all the HROM-DEIM solutions in Fig. 16 relative to the FOM solution. The trend similar to $\mathbf{E r r}_{\text {abs }}$ in Fig. 17 is also found in $\mathbf{E r r}_{\text {msrd }}$. As the number of DEIM cells increases, $\mathbf{E r r}_{\text {msrd }}$ seems to decrease. It is also observed that $\mathbf{E r r}_{\text {msrd }}$ without DEIM exhibits the lowest error compared with those with DEIM. Given 38,400 computational cells in total, even a

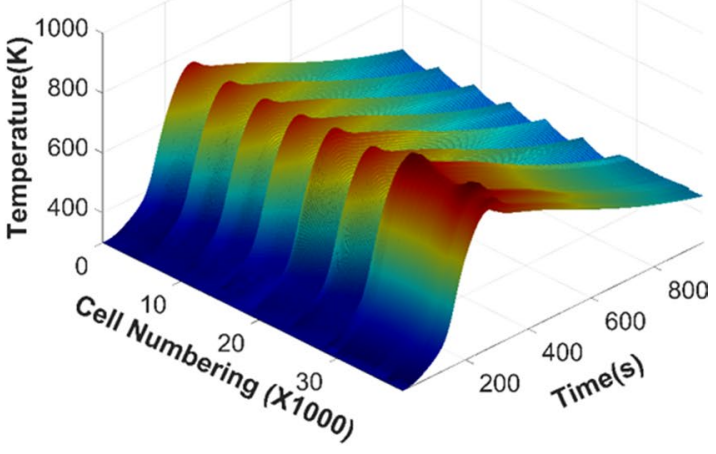

$(b-1)$

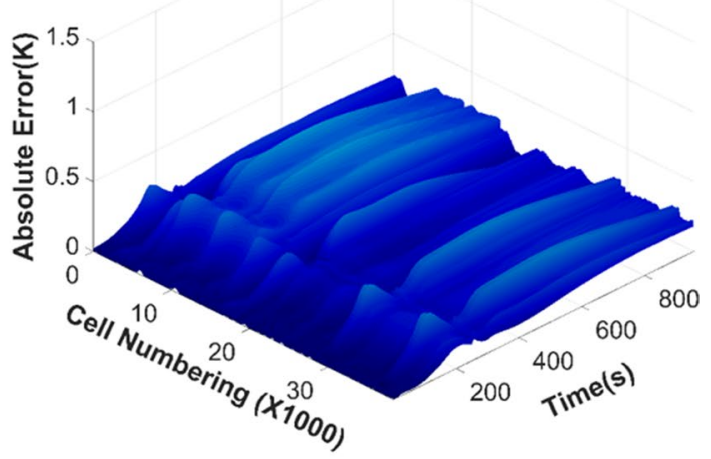

$(\mathrm{b}-2)$

a-1 FROM and b-1 HROM; and the absolute error of a-2 FROM and b-2 HROM relative to FOM

very small number of DEIM cells $\left(m_{A}=50\right)$ for inter-cell transport matrix updating and assembly can yield excellent accuracy (the highest $\mathbf{E r r}_{\text {msrd }} \leq 0.12 \%$ ).

Table 4 lists the computational time of FOM, HROM, and HROM-DEIM in the verification simulation. The time used by HROM is about $33.3 \%$ of FOM, i.e., $66.6 \%$ improvement in simulation speed. Furthermore, with HROMDEIM, more significant computational acceleration can be achieved. Specifically, the computational time ratio of 


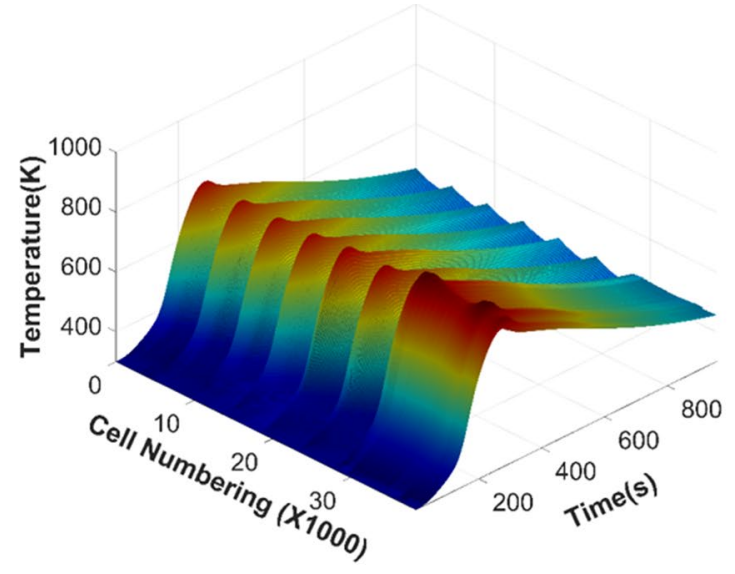

(a)

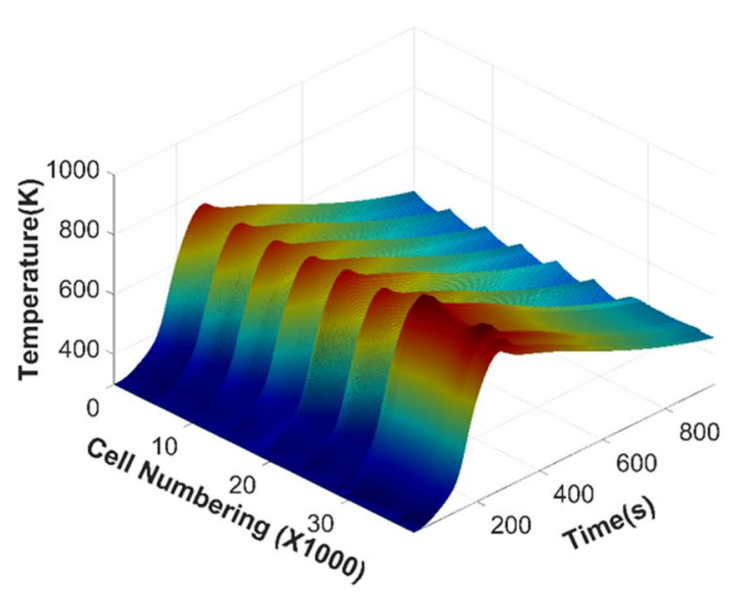

(c)

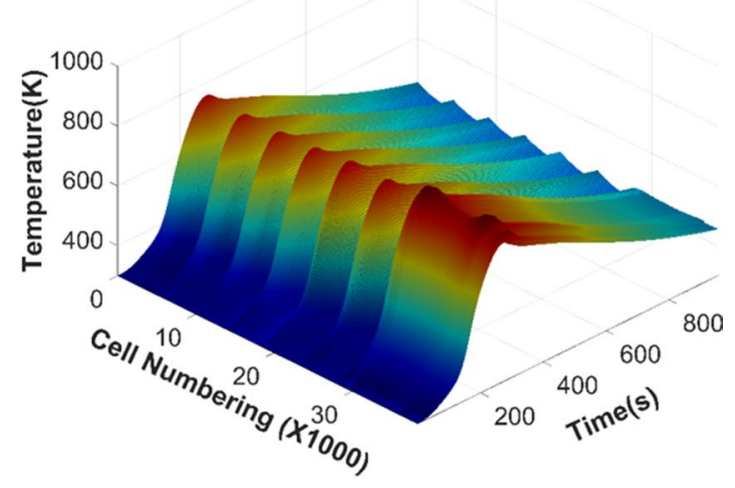

(b)

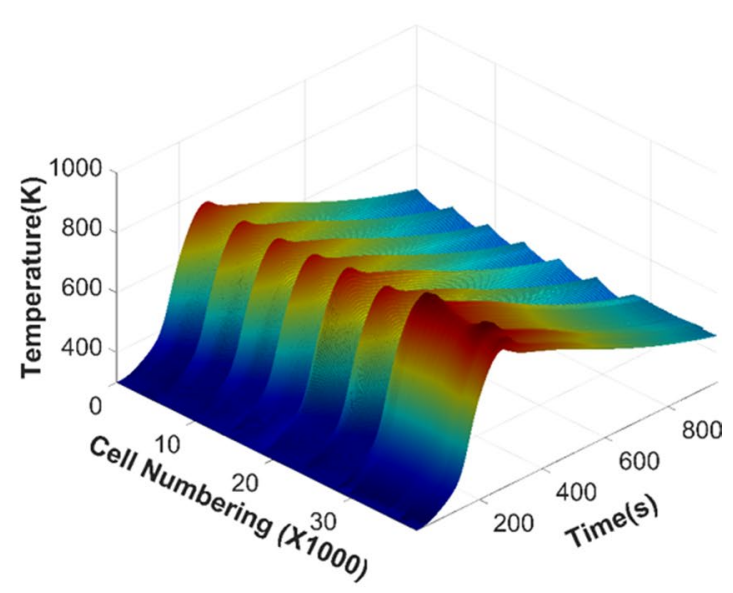

(d)

Fig. 16 The temperature solutions obtained by HROM-DEIM with the different number of DEIM cells in the extended fin array model: a DEIM $50\left(m_{A}=50\right)$, b DEIM $75\left(m_{A}=75\right), \mathbf{c}$ DEIM $100\left(m_{A}=100\right)$ and d non-DEIM

HROM-DEIM to FOM is in the range of $5.48-6.04 \%$ and to HROM is $16.38-18.03 \%$. The studies above indicate that the DEIM interpolation is a viable approach to notably improve ROM efficiency while preserving the simulation accuracy, and the hybrid snapshot simulation could be utilized to construct high-quality HROM-DEIMs with much lower computational efforts/costs.

Similar to the above, the importance of the nonlinearity of the extended fin array model arising from the temperature-dependent thermal conductivity is also inspected. The temperature-dependent thermal conductivity and the constant thermal conductivity (evaluated at the environmental temperature) are given above. Figure 19a shows the temperature solution predicted by FOM using the constant conductivity, and Fig. 19b portrays its difference, i.e., absolute errors $\mathbf{E r r}_{\text {abs }}$, from the FOM results using the temperature-dependent conductivity (not shown to avoid redundant results) for all the 


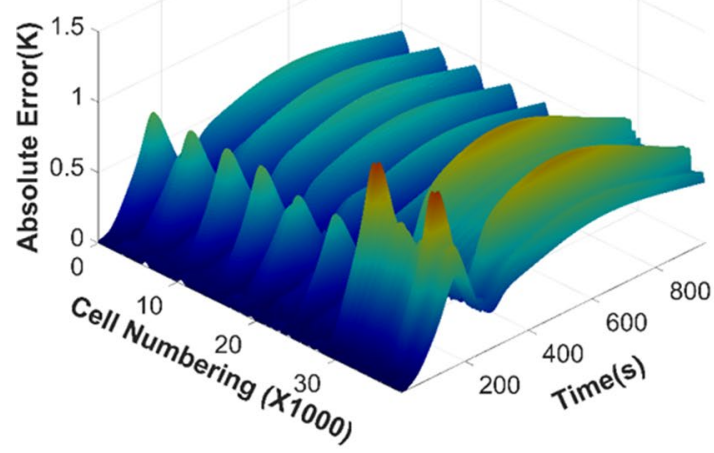

(a)

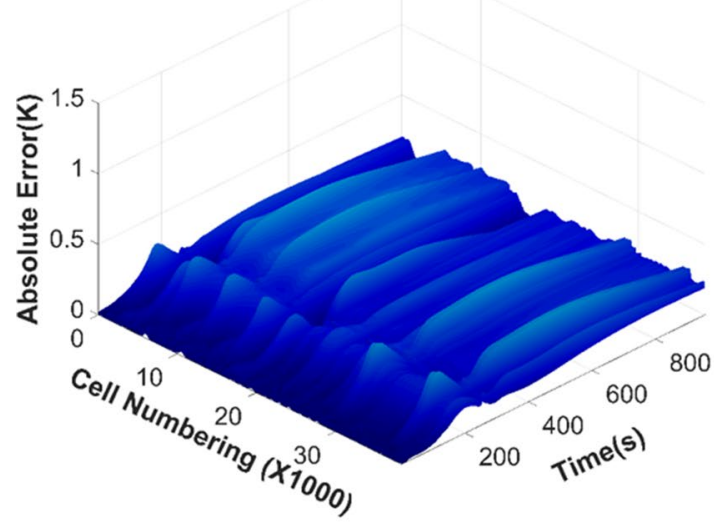

(c)

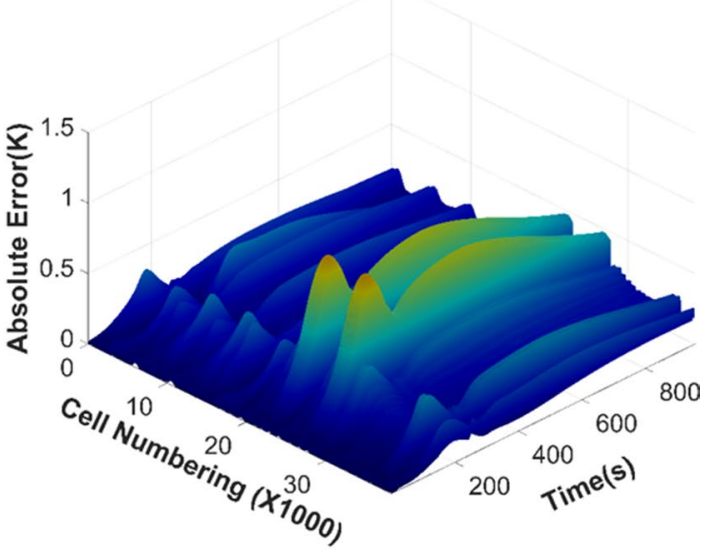

(b)

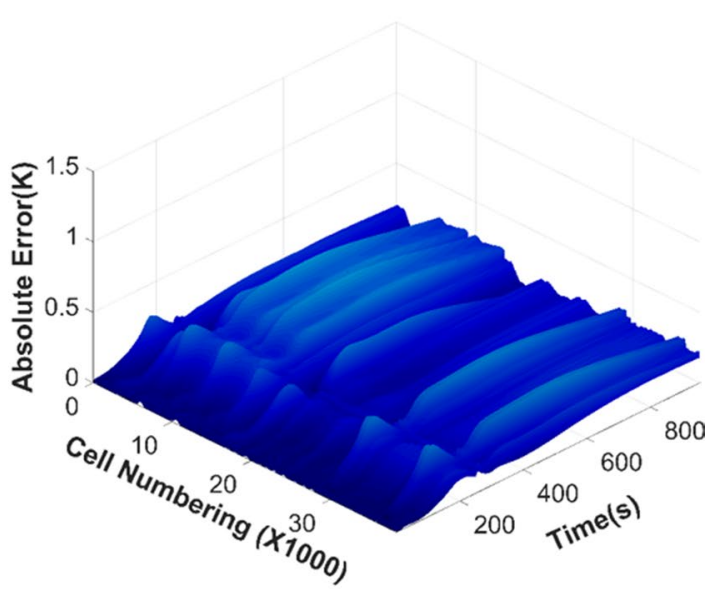

(d)

Fig. 17 The absolute error of the temperature solution obtained by HROM-DEIM with the different number of DEIM cells in the extended fin array model relative to the FOM solution: a DEIM $50\left(m_{A}=50\right), \mathbf{b}$ DEIM $75\left(m_{A}=75\right), \mathbf{c}$ DEIM $100\left(m_{A}=100\right)$ and d non-DEIM

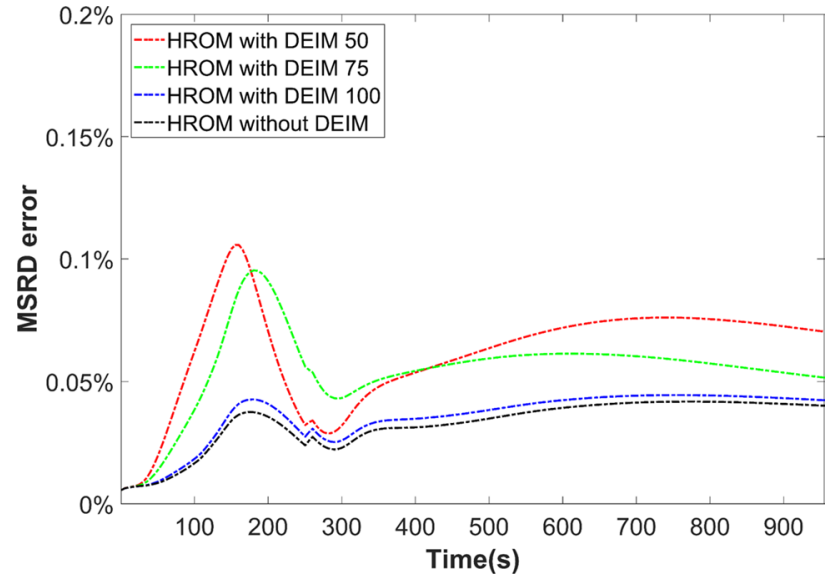

Fig. 18 The MSRD errors versus time for HROM-DEIM with the different number of DEIM cells and HROM without DEIM in the verification simulation in extended fin array model
Table 4 The comparison of computational time in FOM, HROM, and HROM-DEIM in the extended fin array model (Unit:s)

\begin{tabular}{llllll}
\hline Model type & FOM & HROM & \multicolumn{2}{l}{ HROM-DEIM } \\
\cline { 3 - 6 } & & & $m_{A}=50$ & $m_{A}=75$ & $m_{A}=100$ \\
\hline Time & 817.24 & 273.548 & 44.818 & 44.932 & 49.332 \\
\hline
\end{tabular}

computational cells throughout the entire verification simulation. We can see that the maximum value of $\mathbf{E r r}_{\text {abs }}$ goes beyond $60 \mathrm{~K}$, confirming that the aforementioned transport-related nonlinearity of the thermal conduction is important in the model.

\section{SN Applied Sciences}




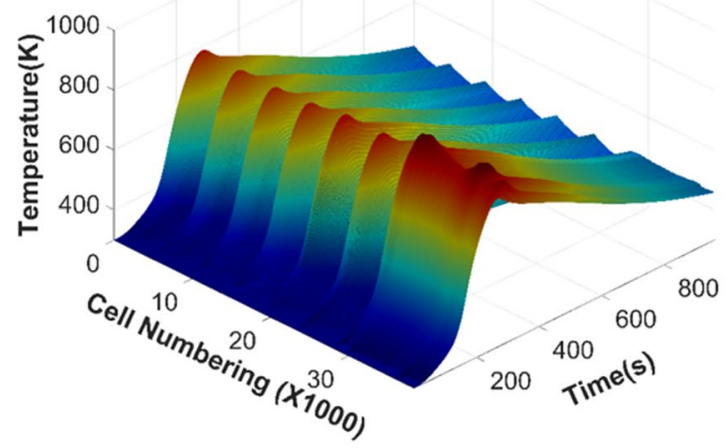

(a)

Fig. 19 Effect of the temperature-dependent thermal conductivity on model nonlinearity: a the temperature solution obtained by FOM in the extended fin array model using the constant thermal

\section{Conclusions}

This paper proposes a methodology to combine the hybrid snapshot simulation and DEIM to reduce the computational cost associated with ROM-DEIM construction and improve computational efficiency of online verification simulation for nonlinear ROMs. The new aspects of the research include: First, an approach is proposed to streamline the hybrid snapshot simulation, snapshot data generation, SVD/iSVD for POD mode construction (both solution variables and nonlinear terms), and ROM-DEIM construction. Second, the method of reconstructing snapshot data of nonlinear terms using ROM solutions is presented to address the issue associated with limited FOM data in the hybrid simulation for enhanced data representation. Last, formulation and data structures are also developed to cast the ROM-DEIM in the hybrid snapshot simulation using the cell-centered FVM scheme to account for transportrelated (non-componentwise) nonlinearity. Key technical findings include:

(1) The feasibility of using the snapshot data generated by hybrid simulation to construct ROM-DEIM is established. That is, the POD modes of the nonlinear terms distilled from the mix of the FOM and reconstructed ROM solution data accurately represent their underlying subspace and can be used to determine DEIM cells/interpolation points.

(2) In the cell-centered FVM formulation, the transport flux at the interface depends on the values of solution variables in the computational cells on both sides. Therefore, to construct ROM-DEIM, the cells adjacent to the DEIM cells/interpolation points also need to

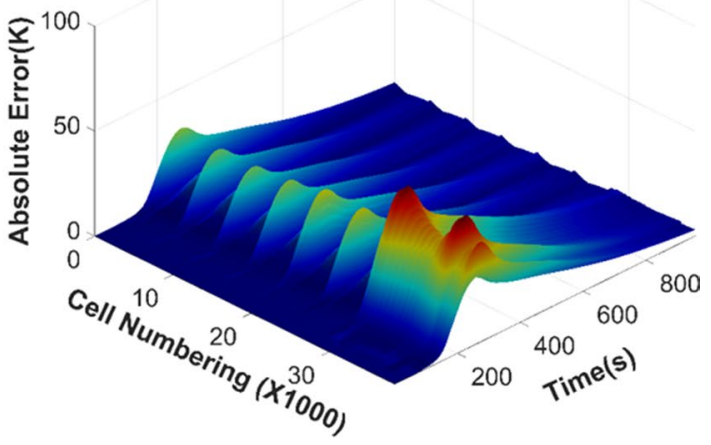

(b)

conductivity; and $\mathbf{b}$ the absolute error between the solution in a and the solution obtained using temperature-dependent thermal conductivity

be extracted in order to update the quantities at the interface and assemble the entries of the nonlinear terms at the DEIM cells.

(3) Following the method proposed in this paper, an accurate global ROM-DEIM is immediately obtained at the end of the hybrid snapshot simulation that can be utilized for fast online verification simulation.

(4) The combination of the hybrid snapshot simulation and ROM-DEIM construction demonstrates excellent computational performance in case studies of numerical heat transfer. Only a small number of intervals, 4 and 2 out of 12, are simulated by FOM in the two case studies, and the computational cost of the snapshot simulation is reduced by $\sim 50 \%$ without compromising accuracy of ROM-DEIM. The performance of the developed ROM-DEIM with the different number of DEIM cells is also evaluated by comparing with the FOM solution for the online verification simulation. MSRD error less than $0.8 \%$ and $0.12 \%$ could be achieved for both case studies. The precomputed POD modes of the nonlinear terms and nonlinear term updating and assembly at the DEIM cells significantly accelerate ROM simulation during the online verification simulation. ROM-DEIM only takes $\sim 5.13 \%$ and $\sim 5.48 \%$ relative to FOM simulation, and $\sim 27.5 \%$ and $\sim 16.38 \%$ relative to HROM without DEIM in both cases.

It should be noted that the ROM-DEIM and hybrid snapshot simulation may be ill-suited for convection-dominated problems that involve moving fronts of the field variables, such as Burgers equation with a shock propagating in a 1D domain. This is because the POD modal 
information and ROM constructed in earlier time intervals may not accurately represent that in the later time intervals, leading to poor approximation, which will be thoroughly investigated in the future. The future work will also include embedding DEIM into the snapshot simulation by developing new model switch criteria to further improve computational efficiency of the hybrid snapshot simulation.

Acknowledgements YW acknowledges the faculty startup grant from the University of South Carolina for partial funding of this research.

\section{Compliance with ethical standards}

Conflict of interest On behalf of all authors, the corresponding author states that there is no conflict of interest.

\section{References}

1. Ali M, Steih K, Urban K (2017) Reduced basis methods with adaptive snapshot computations. Adv Comput Math 43(2):257-294. https://doi.org/10.1007/s10444-016-9485-9

2. Amsallem D, Zahr MJ, Farhat C (2012) Nonlinear model order reduction based on local reduced-order bases. Int J Numer Methods Eng 92(10):891-916. https://doi.org/10.1002/ nme.4371

3. Amsallem D, Zahr MJ, Washabaugh K (2015) Fast local reduced basis updates for the efficient reduction of nonlinear systems with hyper-reduction. Adv Comput Math 41(5):1187-1230. https ://doi.org/10.1007/s10444-015-9409-0

4. Astrid P, Weiland S, Willcox K, Backx T (2008) Missing point estimation in models described by proper orthogonal decomposition. IEEE Trans Autom Control 53(10):2237-2251. https://doi. org/10.1109/TAC.2008.2006102

5. Bai F, Wang Y (2020) Reduced order modeling based on hybrid snapshot simulation. Int J Comput Methods 18:2050029. https ://doi.org/10.1142/S0219876220500292

6. Benner P, Gugercin S, Willcox K (2015) A survey of projectionbased model reduction methods for parametric dynamical systems. SIAM Rev 57(4):483-531. https://doi.org/10.1137/13093 2715

7. Bonomi D, Manzoni A, Quarteroni A (2017) A matrix DEIM technique for model reduction of nonlinear parametrized problems in cardiac mechanics. Comput Methods Appl Mech Eng 324:300-326. https://doi.org/10.1016/j.cma.2017.06.011

8. Brand M (2002) Incremental singular value decomposition of uncertain data with missing values. In: Heyden A, Sparr G, Nielsen M, Johansen P (eds) Computer vision-ECCV 2002. Springer, Berlin, pp 707-720

9. Brand M (2006) Fast low-rank modifications of the thin singular value decomposition. Linear Algebra Appl 415(1):20-30. https ://doi.org/10.1016/j.laa.2005.07.021

10. Bremer J, Goyal P, Feng L, Benner P, Sundmacher K (2017) POD-DEIM for efficient reduction of a dynamic 2D catalytic reactor model. Comput Chem Eng 106:777-784. https://doi. org/10.1016/j.compchemeng.2017.02.032

11. Camphouse RC, Myatt J, Schmit R, Glauser M, Ausseur J, Andino $M$, Wallace R (2008) A snapshot decomposition method for reduced order modeling and boundary feedback control. In: AIAA conference. https://doi.org/10.2514/6.2008-4195
12. Cardoso MA, Durlofsky LJ, Sarma P (2009) Development and application of reduced-order modeling procedures for subsurface flow simulation. Int J Numer Methods Eng 77(9):1322-1350. https://doi.org/10.1002/nme.2453

13. Carlberg K, Farhat C, Cortial J, Amsallem D (2013) The GNAT method for nonlinear model reduction: effective implementation and application to computational fluid dynamics and turbulent flows. J Comput Phys 242:623-647. https://doi. org/10.1016/j.jcp.2013.02.028

14. Chaturantabut S (2017) Temporal localized nonlinear model reduction with a priori error estimate. Appl Numer Math 119:225-238. https://doi.org/10.1016/j.apnum.2017.02.014

15. Chaturantabut $S$, Sorensen DC (2010) Nonlinear model reduction via discrete empirical interpolation. SIAM J Sci Comput 32(5):2737-2764. https://doi.org/10.1137/090766498

16. Chaturantabut $S$, Sorensen DC (2011) Application of POD and DEIM on dimension reduction of non-linear miscible viscous fingering in porous media. Math Comput Model Dyn Syst 17(4):337-353. https://doi.org/10.1080/13873954.2011.547660

17. Chaturantabut $S$, Sorensen DC (2012) A state space error estimate for POD-DEIM nonlinear model reduction. SIAM J Numer Anal 50(1):46-63. https://doi.org/10.1137/110822724

18. Dehghan M, Abbaszadeh M (2018) A combination of proper orthogonal decomposition-discrete empirical interpolation method (POD-DEIM) and meshless local RBF-DQ approach for prevention of groundwater contamination. Comput Math Appl 75(4):1390-1412. https://doi.org/10.1016/j.camwa.2017.11.012

19. Dehghan M, Abbaszadeh $M$ (2018) An upwind local radial basis functions-differential quadrature (RBF-DQ) method with proper orthogonal decomposition (POD) approach for solving compressible Euler equation. Eng Anal Bound Elem 92:244-256. https://doi.org/10.1016/j.enganabound.2017.10.004

20. Fareed H, Singler JR, Zhang Y, Shen J (2018) Incremental proper orthogonal decomposition for PDE simulation data. Comput Math Appl 75(6):1942-1960. https://doi.org/10.1016/j.camwa .2017.09.012

21. Fu X, Nathan Kutz J (2017) Adaptive dimensionality-reduction for time-stepping in differential and partial differential equations. Numer Math Theory Methods Appl 10(4):872-894. https ://doi.org/10.4208/nmtma.2017.m1624

22. Ghavamian F, Tiso P, Simone A (2017) POD-DEIM model order reduction for strain-softening viscoplasticity. Comput Methods Appl Mech Eng 317:458-479. https://doi.org/10.1016/j. cma.2016.11.025

23. Gräßle C, Hinze M (2018) POD reduced-order modeling for evolution equations utilizing arbitrary finite element discretizations. Adv Comput Math 44:1941-1978. https://doi.org/10.1007/s1044 4-018-9620-x

24. Haasdonk B, Ohlberger M (2008) Reduced basis method for finite volume approximations of parametrized linear evolution equations. ESAIM: Math Model Numer Anal 42(2):277-302. https ://doi.org/10.1051/m2an:2008001

25. Hoang K, Khoo B, Liu G, Nguyen N, Patera A (2013) Rapid identification of material properties of the interface tissue in dental implant systems using reduced basis method. Inverse Probl Sci Eng 21(8):1310-1334. https://doi.org/10.1080/17415 977.2012.757315

26. Jørgensen B, Sørensen J, Brøns M (2003) Low-dimensional modeling of a driven cavity flow with two free parameters. Theoret Comput Fluid Dyn 16(4):299-317. https://doi.org/10.1007/s0016 2-002-0082-9

27. Kim T, James DL (2009) Skipping steps in deformable simulation with online model reduction. ACM Trans Graph 28(5):1-9. https ://doi.org/10.1145/1618452.1618469

28. Knezevic DJ, Nguyen NC, Patera AT (2011) Reduced basis approximation and a posteriori error estimation for the parametrized 
unsteady Boussinesq equations. Math Models Methods Appl Sci 21(07):1415-1442. https://doi.org/10.1142/S0218202511005441

29. Mordhorst M, Strecker T, Wirtz D, Heidlauf T, Röhrle O (2017) POD-DEIM reduction of computational EMG models. J Comput Sci 19:86-96. https://doi.org/10.1016/j.jocs.2017.01.009

30. Oxberry GM, Vassilevska TK, Arrighi W, Chand K (2017) Limited memory adaptive snapshot selection for proper orthogonal decomposition. Int J Numer Methods Eng 109(2):198-217. https ://doi.org/10.1002/nme.5283

31. Pando MFD, Schmid PJ, Sipp D (2016) Nonlinear model-order reduction for compressible flow solvers using the discrete empirical interpolation method. J Comput Phys 324:194-209. https://doi.org/10.1016/j.jcp.2016.08.004

32. Peherstorfer B, Willcox K (2015) Dynamic data-driven reducedorder models. Comput Methods Appl Mech Eng 291:21-41. https://doi.org/10.1016/j.cma.2015.03.018

33. Peherstorfer B, Willcox K (2015) Online adaptive model reduction for nonlinear systems via low-rank updates. SIAM J Sci Comput 37(4):A2123-A2150. https://doi.org/10.1137/140989169

34. Peherstorfer B, Willcox K (2016) Data-driven operator inference for nonintrusive projection-based model reduction. Comput Methods Appl Mech Eng 306:196-215. https://doi.org/10.1016/j. cma.2016.03.025

35. Peng L, Mohseni K (2016) Nonlinear model reduction via a locally weighted POD method. Int J Numer Methods Eng 106(5):372-396. https://doi.org/10.1002/nme.5124

36. Qian J, Wang Y, Song H, Pant K, Peabody H, Ku J, Butler CD (2015) Projection-based reduced-order modeling for spacecraft thermal analysis. J Spacecr Rockets 52(3):978-989. https://doi. org/10.2514/1.A33117

37. Rapún ML, Vega JM (2010) Reduced order models based on local POD plus Galerkin projection. J Comput Phys 229(8):3046-3063. https://doi.org/10.1016/j.jcp.2009.12.029

38. Robertson ED, Wang Y, Pant K, Grismer MJ, Camberos JA (2018) A flow feature detection framework for large-scale computational data based on incremental proper orthogonal decomposition and data mining. Int J Comput Fluid Dyn 32(6-7):261-277. https ://doi.org/10.1080/10618562.2018.1508657
39. Sirovich L (1987a) Turbulence and the dynamics of coherent structures. I. Coherent structures. Q Appl Math 45:561-571

40. Sirovich $L$ (1987b) Turbulence and the dynamics of coherent structures. II. Symmetries and transformations. Q Appl Math 45:573-582. https://doi.org/10.1090/qam/910463

41. Sirovich $L$ (1987c) Turbulence and the dynamics of coherent structures. III. Dynamics and scaling. Q Appl Math 45:583-590

42. Terragni F, Valero E, Vega JM (2011) Local POD plus Galerkin projection in the unsteady lid-driven cavity problem. SIAM J Sci Comput 33(6):3538-3561. https://doi.org/10.1137/100816006

43. Ullmann S, Rotkvic M, Lang J (2016) POD-Galerkin reducedorder modeling with adaptive finite element snapshots. J Comput Phys 325:244-258. https://doi.org/10.1016/j.jcp.2016.08.018

44. Vendl A, Faßbender H (2013) Projection-based model order reduction for steady aerodynamics. Springer, Berlin, pp 151166. https://doi.org/10.1007/978-3-642-38877-4_11

45. Wang Y, Song H, Pant K (2014) A reduced-order model for wholechip thermal analysis of microfluidic lab-on-a-chip systems. Microfluid Nanofluid 16(1):369-380. https://doi.org/10.1007/ s10404-013-1210-0

46. Wang Z, McBee B, Iliescu T (2016) Approximate partitioned method of snapshots for POD. J Comput Appl Math 307:374384. https://doi.org/10.1016/j.cam.2015.11.023

47. Willcox K, Peraire J (2002) Balanced model reduction via the proper orthogonal decomposition. AIAA J 40(11):2323-2330

48. Yang YJ, Shen KY (2005) Nonlinear heat-transfer macromodeling for mems thermal devices. J Micromech Microeng 15(2):408

49. Yang YJJ, Kuo CW (2008) Generating scalable and modular macromodels for microchannels using the Galerkin-based technique. IEEE Trans Comput Aided Des Integr Circuits Syst 27(9):1545-1554. https://doi.org/10.1109/TCAD.2008.927764

Publisher's Note Springer Nature remains neutral with regard to jurisdictional claims in published maps and institutional affiliations. 